\title{
Palladium-Catalyzed Coupling of Ammonia and Lithium Amide with Aryl Halides
}

\author{
Qilong Shen and John F. Hartwig* \\ Department of Chemistry, Yale University, \\ P.O. Box 208107, New Haven, Connecticut 06520-8107
}

Supporting Information

General Methods. Unless otherwise noted, all manipulation were conducted under an inert atmosphere. ${ }^{1} \mathrm{H}$ and ${ }^{13} \mathrm{C}$ NMR spectra were recorded on a Bruker DPX 400 or 500 $\mathrm{MHz}$ Spectrometer, and ${ }^{31} \mathrm{P}\left\{{ }^{1} \mathrm{H}\right\}$ NMR spectra were recorded on a General Electric QE $300 \mathrm{MHz}$ spectrometer with tetramethylsilane or residual protiated solvent as a reference. All ${ }^{31} \mathrm{P}\left\{{ }^{1} \mathrm{H}\right\}$ NMR chemical shifts are reported in parts per million relative to an $85 \%$ $\mathrm{H}_{3} \mathrm{PO}_{4}$ external standard. Chemical shifts downfield of the standard are reported with positive values. Elemental analyses were performed by Atlantic Microlabs Inc., Norcross, GA or Robertson Microlab, Inc., Madison, NJ. GC and GC/MS analyses were conducted with an HP-1 methyl silicone column. CyPF- $t$-Bu $(\mathrm{CyPF}-t-\mathrm{Bu}=1$ dicyclohexylphosphino-2-di-t-butylphosphinoethylferrocene) and $\mathrm{Pd}\left(\mathrm{CH}_{3} \mathrm{CN}\right)_{2} \mathrm{Cl}_{2}$ were obtained from Solvias AG and Strem Chemicals and used without further purification. Ethylene glycol dimethyl ether (DME, 99.9\% purity, HPLC grade) was purchased and used without further purification. All other chemicals were used as received from commercial sources.

Synthesis of (CyPF-t-Bu)PdCl $\mathbf{2}_{2}$. Josiphos CyPF-t-Bu (55.4 mg $\left.0.100 \mathrm{mmol}\right)$ was added to a solution of $\mathrm{Pd}\left(\mathrm{CH}_{3} \mathrm{CN}\right)_{2} \mathrm{Cl}_{2}(26.0 \mathrm{mg}, 0.100 \mathrm{mmol})$ in $\mathrm{CH}_{2} \mathrm{Cl}_{2}(5.0 \mathrm{~mL})$. The resulting mixture was stirred for $30 \mathrm{~min}$ at room temperature. The reaction mixture was filtered through a medium fritted funnel containing Celite. The resulting solution was concentrated under vacuum. Crystalline material was obtained by layering with hexane and cooling at $-10{ }^{\circ} \mathrm{C}(65.0 \mathrm{mg}, 90 \%) .{ }^{1} \mathrm{H} \mathrm{NMR}\left(\mathrm{CDCl}_{3}\right) \delta 4.85(\mathrm{~s}, 1 \mathrm{H}), 4.55(\mathrm{~s}, 1 \mathrm{H})$, $4.53(\mathrm{~s}, 1 \mathrm{H}), 4.25(\mathrm{~s}, 5 \mathrm{H}), 3.60-3.75(\mathrm{~m}, 1 \mathrm{H}), 3.00-3.10(\mathrm{~m}, 1 \mathrm{H}), 2.50-2.60(\mathrm{~m}, 1 \mathrm{H})$, 2.27-2.90 (m, $1 \mathrm{H}), 2.13-2.25(\mathrm{~m}, 2 \mathrm{H}), 2.00-2.10(\mathrm{~m}, 1 \mathrm{H}), 1.97(\mathrm{dd}, J=9.0,7.5 \mathrm{~Hz}, 3$ H), 1.70-1.95 (m, 4 H), 1.20-1.30 (m, 8 H), 1.63 (d, J = 13.0 Hz, 9 H), 1.30-1.45 (m, 4 $\mathrm{H}), 1.23(\mathrm{~d}, J=14.5 \mathrm{~Hz}, 9 \mathrm{H}) ;{ }^{31} \mathrm{P}\left\{{ }^{1} \mathrm{H}\right] \mathrm{NMR}\left(\mathrm{CH}_{2} \mathrm{Cl}_{2}\right) \delta 113.83(\mathrm{~d}, J=9.7 \mathrm{~Hz}), 31.78(\mathrm{~d}$, $J=9.7 \mathrm{~Hz}) ;{ }^{13} \mathrm{C} \mathrm{NMR}\left(\mathrm{CDCl}_{3}\right) \delta 96.49(\mathrm{dd}, J=13.3,5.5 \mathrm{~Hz}), 71.92(\mathrm{~d}, J=2.5 \mathrm{~Hz})$, $69.90(\mathrm{~d}, J=9.1 \mathrm{~Hz}), 69.78,69.63(\mathrm{~d}, J=9.2 \mathrm{~Hz}), 69.34(\mathrm{t}, J=5.7 \mathrm{~Hz}), 41.6(\mathrm{~d}, J=35.5$ $\mathrm{Hz}), 41.57(\mathrm{~d}, J=8.2 \mathrm{~Hz}), 40.55(\mathrm{~d}, J=11.2 \mathrm{~Hz}), 37.56(\mathrm{~d}, J=35.5 \mathrm{~Hz}), 34.48(\mathrm{t}, J=9.1$ $\mathrm{Hz}), 31.97$ (d, $J=1.9 \mathrm{~Hz}), 31.05(\mathrm{~d}, J=1.9 \mathrm{~Hz}), 29.99,29.19,28.06,27.55(\mathrm{~d}, J=6.8$ $\mathrm{Hz}), 27.32$ (d, $J=10.2 \mathrm{~Hz}), 26.98$ (d, $J=12.6 \mathrm{~Hz}), 26.89$ (d, $J=5.2 \mathrm{~Hz}), 26.78$ (d, $J=$ $3.8 \mathrm{~Hz}), 26.12(\mathrm{~d}, J=1.9 \mathrm{~Hz}), 25.55,18.02(\mathrm{~d}, J=6.7 \mathrm{~Hz})$. Anal. Calcd. For $\mathrm{C}_{32} \mathrm{H}_{52} \mathrm{Cl}_{2} \mathrm{FeP}_{2} \mathrm{Pd}$ : C, 52.51; H, 7.16. Found: C, 52.72; H, 7.38.

General Procedure for Catalytic Amination of Heteroaryl and Aryl Chlorides, Bromides and Iodides with Ammonia in a Parr Bomb. The reaction conditions and average yields for each reaction are shown in Table 1. A typical procedure is given for the first entry in Table 1.

4-tert-butylaniline (Table 1, entry 1). (CyPF- $t$-Bu) $\mathrm{PdCl}_{2}\left(7.30 \mathrm{mg}, 1.00 \times 10^{-2} \mathrm{mmol}\right)$, $\mathrm{NaO} t \mathrm{Bu}(0.192 \mathrm{~g}, 2.00 \mathrm{mmol})$ and 4-tert-butyl-1-bromobenzene $(0.213 \mathrm{~g}, 1.00 \mathrm{mmol})$ 
were weighed into a Parr Bomb inside a dry box. DME $(20.0 \mathrm{~mL})$ was then added. The Parr bomb was closed and removed from the dry box. Ammonia was added with stirring by connecting to an ammonia tank and maintaining the pressure at $80 \mathrm{psi}$ for $30 \mathrm{~min}$. The resulting reaction mixture was allowed to stir for $24 \mathrm{~h}$ at $90{ }^{\circ} \mathrm{C}$. Pressure was built up to 200 psi during the reaction. The reaction mixture was then cooled to room temperature before being poured into ice water $(20.0 \mathrm{~mL})$. To this mixture was added $\mathrm{HCl}$ aqueous solution $(10.0 \mathrm{~mL}, 1.0 \mathrm{M}))$. The mixture was stirred at room temperature for $5 \mathrm{~min}$ and was then neutralized with a saturated solution of $\mathrm{NaHCO}_{3}(5.00-10.0 \mathrm{~mL})$. After extraction with $\mathrm{CH}_{2} \mathrm{Cl}_{2}(3 \times 20.0 \mathrm{~mL})$, the organic layer was separated and dried over $\mathrm{MgSO}_{4}$. The solvent was evaporated, and the crude product isolated by eluting with hexane/ethyl acetate (70/30) to give $128.1 \mathrm{mg}$ (86\%) of 4-tert-butylaniline as a pale yellow liquid. ${ }^{1} \mathrm{H} \mathrm{NMR}\left(\mathrm{CDCl}_{3}\right) \delta 7.05(\mathrm{~d}, J=8.4 \mathrm{~Hz}, 2 \mathrm{H}), 6.55(\mathrm{~d}, J=8.8 \mathrm{~Hz}, 2 \mathrm{H})$, 3.44 (s, br, $2 \mathrm{H}), 1.20$ (s, $9 \mathrm{H}) ;{ }^{13} \mathrm{C} \mathrm{NMR}\left(\mathrm{CDCl}_{3}\right) \delta 143.74,141.32,125.98,114.86$, $33.85,31.49$.

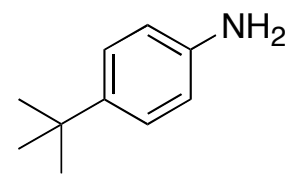

o-Toluidine (Table 1, entry 3). 2-Chlorotoluene (0.126 g, $1.00 \mathrm{mmol})$, (CyPF- $t$ $\mathrm{Bu}) \mathrm{PdCl}_{2}\left(7.30 \mathrm{mg}, 1.00 \times 10^{-2} \mathrm{mmol}\right)$, and $\mathrm{NaO} t \mathrm{Bu}(0.192 \mathrm{~g}, 2.00 \mathrm{mmol})$ in $20.0 \mathrm{~mL}$ DME gave $73.6 \mathrm{mg}(69 \%)$ of $o$-toluidine as a colorless liquid (hexane/ethyl acetate: 70/30). ${ }^{1} \mathrm{H} \mathrm{NMR}\left(\mathrm{CDCl}_{3}\right) \delta 7.08(\mathrm{~d}, J=6.4 \mathrm{~Hz}, 1 \mathrm{H}), 7.07$ (t, $\left.J=6.4 \mathrm{~Hz}, 1 \mathrm{H}\right), 6.74(\mathrm{t}, J=$ $6.0 \mathrm{~Hz}, 1 \mathrm{H}), 6.70(\mathrm{~d}, J=6.0 \mathrm{~Hz}, 1 \mathrm{H}), 3.62(\mathrm{~s}, \mathrm{br}, 2 \mathrm{H}), 2.19(\mathrm{~s}, 3 \mathrm{H}) ;{ }^{13} \mathrm{C} \mathrm{NMR}\left(\mathrm{CDCl}_{3}\right)$ $\delta 144.41,130.36,126.87,122.26,118.57,114.88,17.25$.

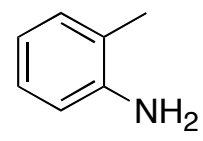

o-Toluidine (Table 1, entry 4). 2-Bromotoluene (0.171 g, $1.00 \mathrm{mmol}$ ), (CyPF- $t$ $\mathrm{Bu}) \mathrm{PdCl}_{2}\left(7.30 \mathrm{mg}, 1.00 \times 10^{-2} \mathrm{mmol}\right)$, and $\mathrm{NaO} t \mathrm{Bu}(0.192 \mathrm{~g}, 2.00 \mathrm{mmol})$ in $20.0 \mathrm{~mL}$ DME gave $89.1 \mathrm{mg}(86 \%)$ of $o$-toluidine as a colorless liquid.

o-Toluidine (Table 1, entry 5). 2-Iodotoluene (0.217 g, $1.00 \mathrm{mmol})$, (CyPF-t$\mathrm{Bu}) \mathrm{PdCl}_{2}\left(7.30 \mathrm{mg}, 1.00 \times 10^{-2} \mathrm{mmol}\right)$, and $\mathrm{NaO} t \mathrm{Bu}(0.192 \mathrm{~g}, 2.00 \mathrm{mmol})$ in $20.0 \mathrm{~mL}$ DME gave $84.7 \mathrm{mg}(79 \%)$ of $o$-toluidine as a colorless liquid.

2-Aminobiphenyl (Table 1, entry 6). 2-Bromobiphenyl (0.233 g, $1.00 \mathrm{mmol})$, (CyPF$t$ - $\mathrm{Bu}) \mathrm{PdCl}_{2}\left(7.30 \mathrm{mg}, 1.00 \times 10^{-2} \mathrm{mmol}\right)$, and $\mathrm{NaO} t \mathrm{Bu}(0.192 \mathrm{~g}, 2.00 \mathrm{mmol})$ in $20.0 \mathrm{~mL}$ DME gave $0.160 \mathrm{~g}(94 \%)$ of 2-aminobiphenyl as a solid (hexane/ethyl acetate: $70 / 30) .{ }^{1} \mathrm{H}$ NMR $\left(\mathrm{CDCl}_{3}\right) \delta$ 7.28-7.34 (m, $\left.4 \mathrm{H}\right), 7.19-7.25(\mathrm{~m}, 1 \mathrm{H}), 6.98-7.07(\mathrm{~m}, 2 \mathrm{H}), 6.71(\mathrm{t}, J=$ $7.2 \mathrm{~Hz}, 1 \mathrm{H}), 6.62(\mathrm{~d}, J=8.0 \mathrm{~Hz}, 1 \mathrm{H}), 3.60(\mathrm{~s}, \mathrm{br}, 2 \mathrm{H}) ;{ }^{13} \mathrm{C} \mathrm{NMR}\left(\mathrm{CDCl}_{3}\right) \delta 140.40$, $139.44,130.36,129.00,128.71,128.41,127.52,127.06,118.54,115.50$. 
<smiles>Nc1ccccc1-c1ccccc1</smiles>

2-iso-Propylaniline (Table 1, entry 7). 1-Bromo-2-iso-propylbenzene (0.199 g, 1.00 mmol), (CyPF- $t$-Bu) $\mathrm{PdCl}_{2}\left(7.30 \mathrm{mg}, 1.00 \times 10^{-2} \mathrm{mmol}\right)$, and $\mathrm{NaO} t \mathrm{Bu}(0.192 \mathrm{~g}, 2.00$ $\mathrm{mmol}$ ) in $4.0 \mathrm{~mL}$ DME gave $0.120 \mathrm{~g}(89 \%)$ of 2-iso-propylaniline as a solid (hexane/ethyl acetate: 70/30). ${ }^{1} \mathrm{H}$ NMR $\left(\mathrm{CDCl}_{3}\right) \delta 7.08(\mathrm{dd}, J=6.0,1.2 \mathrm{~Hz}, 1 \mathrm{H}), 6.96$ $(\mathrm{td}, J=6.0,1.2 \mathrm{~Hz}, 1 \mathrm{H}), 6.73(\mathrm{td}, J=6.0,0.8 \mathrm{~Hz}, 1 \mathrm{H}), 6.60(\mathrm{dd}, J=6.0,1.2 \mathrm{~Hz}, 1 \mathrm{H})$, 3.56 (s, br, $2 \mathrm{H}), 2.83$ (sept, $J=5.2 \mathrm{~Hz}, 1 \mathrm{H}), 1.20(J=5.2 \mathrm{~Hz}, 6 \mathrm{H}) ;{ }^{13} \mathrm{C} \mathrm{NMR}\left(\mathrm{CDCl}_{3}\right) \delta$ $143.22,132.55,126.44,125.30,118.92,115.74,27.56,22.20$.<smiles>CC(C)c1ccccc1N</smiles>

4-Amino-iso-quinoline (Table 1, entry 8). 4-Bromo-iso-quinoline (0.208 g, 1.00 mmol), (CyPF- $t$-Bu) $\mathrm{PdCl}_{2}\left(7.30 \mathrm{mg}, 1.00 \times 10^{-2} \mathrm{mmol}\right)$, and $\mathrm{NaO} t \mathrm{Bu}(0.192 \mathrm{~g}, 2.00$ $\mathrm{mmol})$ in $20.0 \mathrm{~mL}$ DME gave $0.115 \mathrm{~g}(80 \%)$ of 4-amino-iso-quinoline as a solid Ethyl acetate/methanol: 50/50). ${ }^{1} \mathrm{H}$ NMR $\left(\mathrm{CDCl}_{3}\right) \delta 8.70(\mathrm{~s}, 1 \mathrm{H}), 8.00(\mathrm{~s}, 1 \mathrm{H}), 7.84(\mathrm{~d}, J=8.0$ $\mathrm{Hz}, 1 \mathrm{H}), 7.76(\mathrm{~d}, J=8.5 \mathrm{~Hz}, 1 \mathrm{H}), 7.58(\mathrm{dd}, J=8.0,7.0 \mathrm{~Hz}, 1 \mathrm{H}), 7.51(\mathrm{dd}, J=8.0,7.5$ $\mathrm{Hz}, 1 \mathrm{H}), 4.21(\mathrm{~s}, 2 \mathrm{H}) ;{ }^{13} \mathrm{C} \mathrm{NMR}\left(\mathrm{CDCl}_{3}\right) \delta 142.99,136.86,128.86,128.54,127.99$, $127.65,126.93,125.95,119.97$.<smiles>Nc1cncc2ccccc12</smiles>

5-Amino-iso-quinoline (Table 1, entry 9). 5-Bromo-iso-quinoline (0.208 g, 1.00 mmol), (CyPF- $t$-Bu) $\mathrm{PdCl}_{2}\left(7.30 \mathrm{mg}, 1.00 \times 10^{-2} \mathrm{mmol}\right)$, and $\mathrm{NaO} t \mathrm{Bu}(0.192 \mathrm{~g}, 2.00$ $\mathrm{mmol}$ ) in $4.0 \mathrm{~mL}$ DME gave $0.101 \mathrm{~g}(70 \%)$ of 5-Amino-iso-quinoline as a solid (Ethyl acetate/methanol: 90/10). ${ }^{1} \mathrm{H}$ NMR $\left(\mathrm{CDCl}_{3}\right) \delta 9.15(\mathrm{~s}, 1 \mathrm{H}), 8.45(\mathrm{~d}, J=5.6 \mathrm{~Hz}, 1 \mathrm{H})$, $7.55(\mathrm{~d}, J=6.0 \mathrm{~Hz}, 1 \mathrm{H}), 7.37(\mathrm{~d}, J=5.2 \mathrm{~Hz}, 2 \mathrm{H}), 6.91(\mathrm{t}, J=4.8 \mathrm{~Hz}, 1 \mathrm{H}), 4.26$ (s, br, 2 $\mathrm{H}) ;{ }^{13} \mathrm{C} \mathrm{NMR}\left(\mathrm{CDCl}_{3}\right) \delta 152.85,141.88,141.30,129.33,127.72,125.88,117.80,114.06$, 112.96 .<smiles>Nc1cccc2cnccc12</smiles>

1-Aminonaphthalene (Table 1, entry 10). 1-Bromonapthathene (0.207 g, $1.00 \mathrm{mmol})$, $(\mathrm{CyPF}-t-\mathrm{Bu}) \mathrm{PdCl}_{2}\left(7.30 \mathrm{mg}, 1.00 \times 10^{-2} \mathrm{mmol}\right)$, and $\mathrm{NaO} t \mathrm{Bu}(0.192 \mathrm{~g}, 2.00 \mathrm{mmol})$ in 4.0 
$\mathrm{mL}$ DME gave $0.132 \mathrm{~g}$ (92\%) of 1-Aminonaphthalene as a solid (hexane/ethyl acetate: 70/30). ${ }^{1} \mathrm{H}$ NMR $\left(\mathrm{CDCl}_{3}\right) \delta$ 7.78-7.82 (m, $\left.2 \mathrm{H}\right), 7.40-7.7 .47(\mathrm{~m}, 2 \mathrm{H})$, 7.25-7.34 (m, $\left.2 \mathrm{H}\right)$, $6.76(\mathrm{dd}, J=6.8,1.2 \mathrm{~Hz}, 1 \mathrm{H}), 4.10(\mathrm{~s}, \mathrm{br}, 2 \mathrm{H}) ;{ }^{13} \mathrm{C} \mathrm{NMR}\left(\mathrm{CDCl}_{3}\right) \delta 140.02,134.32$, $128.49,126.29,125.79,124.80,123.58,120.74,118.90,109.62$.<smiles>Nc1cccc2ccccc12</smiles>

General Procedure for Catalytic Amination of Heteroaryl and Aryl Chlorides, Bromides and Iodides with Lithium Amide $\left(\mathbf{L i N H}_{2}\right)$. The reaction conditions and average yields for each reaction are shown in 2. A typical procedure is given for the first entry in Table 2.

4-tert-butylaniline (Table 2, entry 1). (CyPF-t-Bu) $\mathrm{PdCl}_{2}\left(7.30 \mathrm{mg}, 1.00 \times 10^{-2} \mathrm{mmol}\right)$, $\mathrm{LiNH}_{2}(0.230 \mathrm{~g}, 10.0 \mathrm{mmol})$ and 4-tert-butyl-1-bromobenzene $(0.213 \mathrm{~g}, 1.00 \mathrm{mmol})$ were weighed into a $24 \mathrm{~mL}$ vial. DME $(20.0 \mathrm{~mL})$ was then added. The vial was sealed with a cap containing a PTFE septum, and the reaction mixture was stirred for $24 \mathrm{~h}$ at $80{ }^{\circ} \mathrm{C}$. The reaction mixture was allowed to cool to room temperature before pouring into ice water $(20.0 \mathrm{~mL})$. To this mixture was added $\mathrm{HCl}$ aqueous solution $(10.0 \mathrm{~mL}, 1.0 \mathrm{M})$. The mixture was stirred at room temperature for $5 \mathrm{~min}$ and was then neutralized with a saturated solution of $\mathrm{NaHCO}_{3}(5.00-10.0 \mathrm{~mL})$. After extraction with $\mathrm{CH}_{2} \mathrm{Cl}_{2}(3 \times 20.0$ $\mathrm{mL}$ ), the organic layer was separated and dried over $\mathrm{MgSO}_{4}$. The solvent was evaporated, and the crude product isolated by eluting with hexane/ethyl acetate (80/20) to give 107.0 $\mathrm{mg}(72 \%)$ of 4-tert-butylaniline as a pale yellow liquid.

$\boldsymbol{o}$-Toluidine (Table 2, entry 4). 2-Chlorotoluene (0.126 g, $1.00 \mathrm{mmol}$ ), (CyPF- $t$ $\mathrm{Bu}) \mathrm{PdCl}_{2}\left(7.30 \mathrm{mg}, 1.00 \times 10^{-2} \mathrm{mmol}\right)$, and $\mathrm{LiNH}_{2}(0.230 \mathrm{~g}, 10.0 \mathrm{mmol})$ in $20.0 \mathrm{~mL} \mathrm{DME}$ gave $74.9 \mathrm{mg}(70 \%)$ of $o$-toluidine as a colorless liquid.

o-Toluidine (Table 2, entry 5). 2-Bromotoluene (0.171 g, $1.00 \mathrm{mmol}$ ), (CyPF-t$\mathrm{Bu}) \mathrm{PdCl}_{2}\left(7.30 \mathrm{mg}, 1.00 \times 10^{-2} \mathrm{mmol}\right)$, and $\mathrm{LiNH}_{2}(0.230 \mathrm{~g}, 10.0 \mathrm{mmol})$ in $20.0 \mathrm{~mL} \mathrm{DME}$ gave $89.5 \mathrm{mg}(86 \%)$ of $o$-toluidine as a colorless liquid.

o-Toluidine (Table 2, entry 6). 2-Iodotoluene (0.217 g, $1.00 \mathrm{mmol}$ ), (CyPF-t$\mathrm{Bu}) \mathrm{PdCl}_{2}\left(7.30 \mathrm{mg}, 1.00 \times 10^{-2} \mathrm{mmol}\right)$, and $\mathrm{LiNH}_{2}(0.230 \mathrm{~g}, 10.0 \mathrm{mmol})$ in $20.0 \mathrm{~mL} \mathrm{DME}$ gave $86.8 \mathrm{mg}(81 \%)$ of $o$-toluidine as a colorless liquid.

2-Aminobiphenyl (Table 2, entry 7). 2-Bromobiphenyl (0.233 g, $1.00 \mathrm{mmol})$, (CyPF$t$-Bu $) \mathrm{PdCl}_{2}\left(7.30 \mathrm{mg}, 1.00 \times 10^{-2} \mathrm{mmol}\right)$, and $\mathrm{LiNH}_{2}(0.230 \mathrm{~g}, 10.0 \mathrm{mmol})$ in $20.0 \mathrm{~mL}$ DME gave $0.129 \mathrm{~g}(76 \%)$ of 2-Aminobiphenyl as a solid.

2-iso-Propylaniline (Table 2, entry 8). 1-Bromo-2-iso-propylbenzene (0.199 g, 1.00 $\mathrm{mmol}),(\mathrm{CyPF}-t-\mathrm{Bu}) \mathrm{PdCl}_{2}\left(7.30 \mathrm{mg}, 1.00 \times 10^{-2} \mathrm{mmol}\right)$, and $\mathrm{LiNH}_{2}(0.230 \mathrm{~g}, 10.0 \mathrm{mmol})$ in $2.0 \mathrm{~mL}$ DME gave $0.109 \mathrm{~g}(81 \%)$ of 2-iso-Propylaniline as a solid.

4-Amino-iso-quinoline (Table 2, entry 9). 4-Bromo-iso-quinoline (0.208 g, 1.00 $\mathrm{mmol}),(\mathrm{CyPF}-t-\mathrm{Bu}) \mathrm{PdCl}_{2}\left(7.30 \mathrm{mg}, 1.00 \times 10^{-2} \mathrm{mmol}\right)$, and $\mathrm{LiNH}_{2}(0.230 \mathrm{~g}, 10.0 \mathrm{mmol})$ in $20.0 \mathrm{~mL}$ DME gave $0.118 \mathrm{~g}(82 \%)$ of 4-Amino-iso-quinoline as a solid.

5-Amino-iso-quinoline (Table 2, entry 10). 5-Bromo-iso-quinoline (0.208 g, 1.00 $\mathrm{mmol}),(\mathrm{CyPF}-t-\mathrm{Bu}) \mathrm{PdCl}_{2}\left(7.30 \mathrm{mg}, 1.00 \times 10^{-2} \mathrm{mmol}\right)$, and $\mathrm{LiNH}_{2}(0.230 \mathrm{~g}, 10.0 \mathrm{mmol})$ in $2.0 \mathrm{~mL} \mathrm{DME}$ gave $0.114 \mathrm{~g}(79 \%)$ of 5-Amino-iso-quinoline as a solid. 
1-Aminonaphthalene (Table 2, entry 11). 1-Bromonapthathene $(0.207 \mathrm{~g}, 1.00 \mathrm{mmol})$, $(\mathrm{CyPF}-t-\mathrm{Bu}) \mathrm{PdCl}_{2}\left(7.30 \mathrm{mg}, 1.00 \times 10^{-2} \mathrm{mmol}\right)$, and $\mathrm{LiNH}_{2}(0.230 \mathrm{~g}, 10.0 \mathrm{mmol})$ in 4.0 $\mathrm{mL}$ DME gave $0.128 \mathrm{~g}(89 \%)$ of 1-Aminonaphthalene as a solid.

2-Aminonaphthalene (Table 2, entry 12). 2-Bromonapthathene (0.207 g, $1.00 \mathrm{mmol})$, $(\mathrm{CyPF}-t-\mathrm{Bu}) \mathrm{PdCl}_{2}\left(7.30 \mathrm{mg}, 1.00 \times 10^{-2} \mathrm{mmol}\right)$, and $\mathrm{LiNH}_{2}(0.230 \mathrm{~g}, 10.0 \mathrm{mmol})$ in 4.0 $\mathrm{mL}$ DME gave $0.100 \mathrm{~g}(69 \%)$ of 1-Aminonaphthalene as a solid (hexane/ethyl acetate: 70/30). ${ }^{1} \mathrm{H}$ NMR $\left(\mathrm{CDCl}_{3}\right) \delta 7.67(\mathrm{~d}, J=8.4 \mathrm{~Hz}, 1 \mathrm{H}), 7.63(\mathrm{~d}, J=8.4 \mathrm{~Hz}, 1 \mathrm{H}), 7.57(\mathrm{~d}, J$ $=8.0 \mathrm{~Hz}, 1 \mathrm{H}), 7.35(\mathrm{td}, J=7.2,1.2 \mathrm{~Hz}, 1 \mathrm{H}), 7.21(\mathrm{td}, J=7.2,1.6 \mathrm{~Hz}, 1 \mathrm{H}), 6.93(\mathrm{~s}, 1$ $\mathrm{H}), 6.90(\mathrm{dd}, J=8.4,2.0 \mathrm{~Hz}, 1 \mathrm{H}), 3.76(\mathrm{~s}, \mathrm{br}, 2 \mathrm{H}) ;{ }^{13} \mathrm{C} \mathrm{NMR}\left(\mathrm{CDCl}_{3}\right) \delta 144.05,134.83$, $129.12,127.86,127.65,126.27,125.73,122.38,118.17,108.48$.<smiles>Nc1ccc2ccccc2c1</smiles>

1-Amino-4-chlorobenzene (Table 2, entry 13). 1-Bromo-4-chlorobenzene $(0.207 \mathrm{~g}$, $1.00 \mathrm{mmol}),(\mathrm{CyPF}-t-\mathrm{Bu}) \mathrm{PdCl}_{2}\left(7.30 \mathrm{mg}, 1.00 \times 10^{-2} \mathrm{mmol}\right)$, and $\mathrm{LiNH}_{2}(0.230 \mathrm{~g}, 10.0$ $\mathrm{mmol})$ in $2.0 \mathrm{~mL}$ DME gave $86.7 \mathrm{mg}(68 \%)$ of 1-Amino-4-chlorobenzene as a solid (hexane/ethyl acetate: 70/30). ${ }^{1} \mathrm{H}$ NMR $\left(\mathrm{CDCl}_{3}\right) \delta 7.08(\mathrm{~d}, J=8.4 \mathrm{~Hz}, 2 \mathrm{H}), 6.58(\mathrm{~d}, J=$ $8.8 \mathrm{~Hz}, 2 \mathrm{H}), 3.63$ (s, br, $2 \mathrm{H}) ;{ }^{13} \mathrm{C} \mathrm{NMR}\left(\mathrm{CDCl}_{3}\right) \delta$ 144.90, 129.05, 123.05, 116.17.<smiles>Nc1ccc(Cl)cc1</smiles>

Carbazole (Table 2, entry 14). 2,2'-Dibromobiphenyl (0.207 g, $1.00 \mathrm{mmol})$, (CyPF- $t$ $\mathrm{Bu}) \mathrm{PdCl}_{2}\left(7.30 \mathrm{mg}, 1.00 \times 10^{-2} \mathrm{mmol}\right)$, and $\mathrm{LiNH}_{2}(0.230 \mathrm{~g}, 10.0 \mathrm{mmol})$ in $2.0 \mathrm{~mL} \mathrm{DME}$ gave $0.107 \mathrm{~g}(64 \%)$ of Carbazole as a solid (hexane/ethyl acetate: 70/30). ${ }^{1} \mathrm{H}$ NMR $\left(\mathrm{CDCl}_{3}\right) \delta 8.08(\mathrm{~d}, J=7.6 \mathrm{~Hz}, 2 \mathrm{H}), 7.99(\mathrm{~s}, \mathrm{br}, 1 \mathrm{H}), 7.39-7.44(\mathrm{~m}, 4 \mathrm{H}), 7.21-7.27$ (m, 2 $\mathrm{H}) ;{ }^{13} \mathrm{C}$ NMR $\left(\mathrm{CDCl}_{3}\right) \delta 139.43,125.81,123.31,120.31,119.41,110.54$.

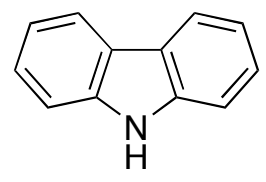

Procedure for Large-Scale Catalytic Amination of 1-Bromo-2-iso-propyl benzene with Lithium Amide (CyPF- $t$-Bu) $\mathrm{PdCl}_{2}(73.0 \mathrm{mg}, 100 \mathrm{mmol}), \mathrm{LiNH}_{2}(2.30 \mathrm{~g}, 100$ $\mathrm{mmol}$ ) and 1-Bromo-2-iso-propylbenzene $(1.99 \mathrm{~g}, 100 \mathrm{mmol})$ were weighed into a 100 $\mathrm{mL}$ round bottom flask with a stirring bar. DME $(20.0 \mathrm{~mL})$ was then added. The flask was sealed with a cap and wrapped tightly with electrical tape. The reaction mixture was stirred for $24 \mathrm{~h}$ at $90{ }^{\circ} \mathrm{C}$. The reaction mixture was allowed to cool to room temperature before pouring into ice water $(50.0 \mathrm{~mL})$. To this mixture was added aqueous $\mathrm{HCl}(100$ $\mathrm{mL}, 1.0 \mathrm{M}$ ). The mixture was stirred at room temperature for $5 \mathrm{~min}$ and was then neutralized with a saturated solution of $\mathrm{NaHCO}_{3}(50.0 \mathrm{~mL})$. After extraction with $\mathrm{CH}_{2} \mathrm{Cl}_{2}$ $(3 \times 50.0 \mathrm{~mL})$, the organic layer was separated and dried over $\mathrm{MgSO}_{4}$. The solvent was 
evaporated, and the crude product isolated by column chromatography, eluting with hexane/ethyl acetate (70/30) to give $1.11 \mathrm{~g} \mathrm{(82 \% )}$ of 2-iso-propylaniline as a solid.

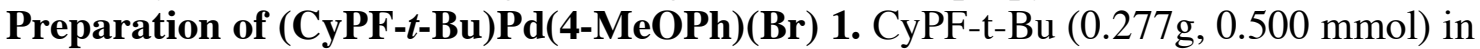
$2.0 \mathrm{~mL}$ benzene was added to a mixture of $\mathrm{Pd}\left[\mathrm{P}(o-\mathrm{Tol})_{3}\right]_{2}(0.357 \mathrm{~g}, 0.500 \mathrm{mmol})$ and $4-$ bromoanisole $(0.467 \mathrm{~g}, 5.00 \mathrm{mmol})$ in $20 \mathrm{ml}$ benzene. The mixture was allowed to stir at room temperature for $30 \mathrm{~min}$. The resulting red solution was filtered, and the solvent was evaporated under vacuum. The residue was redissolved in $2 \mathrm{~mL}$ THF. The THF solution was layered with pentane and cooled at $-10{ }^{\circ} \mathrm{C}$. The product was deposited as red crystals. The crystals were filtered and washed with pentane and dried under vacuum to give $356 \mathrm{mg}(84 \%)$ of a single isomer of the product. ${ }^{1} \mathrm{H} \mathrm{NMR}\left(\mathrm{CDCl}_{3}\right) \delta 0.80-2.80(\mathrm{~m}$, $22 \mathrm{Cy} \mathrm{H}), 1.16(\mathrm{~d}, J=12.8 \mathrm{~Hz}, 9 \mathrm{H}), 1.66(\mathrm{~d}, J=11.6 \mathrm{~Hz}, 9 \mathrm{H}), 1.80-1.84$ (m, $4 \mathrm{H}$ from THF), 1.96 (t, $J=7.6 \mathrm{~Hz}, 3 \mathrm{H}), 3.17$ (dq, $J=6.0,5.6 \mathrm{~Hz}, 1 \mathrm{H}), 3.74$ (s, $3 \mathrm{H}), 3.71-3.76$ $(\mathrm{m}, 4 \mathrm{H}$ from THF), $4.23(\mathrm{~s}, 5 \mathrm{H}), 4.30(\mathrm{t}, J=2.4 \mathrm{~Hz}, 1 \mathrm{H}), 4.49(\mathrm{~s}, \mathrm{br}, 1 \mathrm{H}), 4.85$ (s, br, 1 H), $6.55(\mathrm{~d}, J=7.2 \mathrm{~Hz}, 1 \mathrm{H}), 6.77(\mathrm{~d}, J=6.8 \mathrm{~Hz}, 1 \mathrm{H}), 7.10-7.25$ (s, br, $1 \mathrm{H}), 7.25-7.40$ (s, br, $1 \mathrm{H}) ;{ }^{31} \mathrm{P}\left\{{ }^{1} \mathrm{H}\right] \mathrm{NMR}\left(\mathrm{CH}_{2} \mathrm{Cl}_{2},-30{ }^{\circ} \mathrm{C}\right) 72.83(\mathrm{~d}, J=34.5 \mathrm{~Hz}), 18.08(\mathrm{~d}, J=34.8$ $\mathrm{Hz}$ ); Anal. Calcd. For $\mathrm{C}_{43} \mathrm{H}_{67} \mathrm{BrFeO}_{2} \mathrm{P}_{2} \mathrm{Pd} \bullet \mathrm{THF}$ : C, 56.13; H, 7.34. Found: C, 56.35; H, 7.65 .

Stoichiometric reactions of $(\mathrm{CyPF}-t-\mathrm{Bu}) \mathrm{Pd}(4-\mathrm{MeOPh})(\mathrm{Br})^{8}(1)$ with ammonia and NaOtBu. (CyPF- $t$-Bu)Pd(4-MeOPh)(Br) $\left(8.2 \mathrm{mg}, 1.0 \times 10^{-3} \mathrm{mmol}\right), \mathrm{NaO} t \mathrm{Bu}(1.0 \mathrm{mg}, 1.0$ $\left.\times 10^{-3} \mathrm{mmol}\right)$ and $\mathrm{P}\left(\mathrm{Ph}-d_{5}\right)_{3}\left(2.7 \mathrm{mg}, 2.0 \times 10^{-3} \mathrm{mmol}\right)$ were dissolved $0.5 \mathrm{~mL} \mathrm{C}_{6} \mathrm{D}_{6}$ containing 1,3,5-trimethoxybenzene $\left(0.29 \mathrm{mg}, 1.7 \times 10^{-3} \mathrm{mmol}\right)$. A ${ }^{1} \mathrm{H}$ NMR spectrum was recorded. $\mathrm{NH}_{3}\left(5.0 \mathrm{~cm}^{3}\right.$ at 0.1 atm pressure $)$ was added by vacuum transfer. The tube was sealed and heated at $90{ }^{\circ} \mathrm{C}$ in a oil bath. ${ }^{31} \mathrm{P}\left\{{ }^{1} \mathrm{H}\right\}$ NMR spectrometry showed the disappearance of the starting material and the formation of (CyPF-t-Bu) Pd P(Ph- $\left.d_{5}\right)_{3}$. A ${ }^{1} \mathrm{H}$ NMR spectrum was recorded, and the yield of 4-tert-butylaniline and di(4-tertbutylphenyl)amine was determined by the integrating the aromatic $\mathrm{C}-\mathrm{H}$ resonances of amines vs. the internal standard.

Preparation of $\left[(\mathrm{CyPF}-t-\mathrm{Bu}) \mathrm{Pd}(4-\mathrm{MeOPh})\left(\mathrm{NH}_{3}\right)\right] \mathrm{OTf} \quad$ (2). (CyPF- $t$-Bu)Pd(4$\mathrm{MeOPh})(\mathrm{Br}) 1(220 \mathrm{mg}, 25.9 \mathrm{mmol})$ was dissolved in $5 \mathrm{~mL}$ of $\mathrm{CH}_{2} \mathrm{Cl}_{2}$ in a reaction tube sealed to a high vacuum valve. $50 \mathrm{~cm}^{3}$ of ammonia at $0.3 \mathrm{~atm}$ pressure was condensed into the reaction tube by vacuum transfer. The tube was brought into the dry box AgOTf (66.0mg, $25.9 \mathrm{mmol}$ ) was added. The solution turned cloudy immediately. After stirring at room temperature for $30 \mathrm{~min}$, the solution was carefully decanted and filtered through a plug of Celite. The resulting solution was evaporated to approximately $1 / 10^{\text {th }}$ of the original volume. The solution was layered with toluene and allowed to stand at $-35{ }^{\circ} \mathrm{C}$ overnight. The product was isolated as a pale yellow powder. Crystals suitable for X-ray diffraction was obtained by slow crystallization of a toluene solution of the complex at room temperature. ${ }^{1} \mathrm{H}$ NMR $\left(\mathrm{THF}-d_{8},-30{ }^{\circ} \mathrm{C}\right) \delta$ 7.48-7.56 $(\mathrm{m}, 1 \mathrm{H}), 7.05-7.22(\mathrm{~m}, 1 \mathrm{H})$, 7.05-7.22 (m, from toluene), 6.80-6.90 (m, $1 \mathrm{H}), 6.65-6.69(\mathrm{~m}, 1 \mathrm{H}), 5.07(\mathrm{~s}, \mathrm{br}, 0.74 \mathrm{H}$, major), 4.96 (s, br, 0.20 H from minor), 4.83 (s, br, $0.78 \mathrm{H}$ from major), 4,68 (s, br, 0.27 $\mathrm{H}$ from minor), 4.63 (s, br, $1 \mathrm{H}), 4.27$ (s, $1 \mathrm{H}$ from minor), 4.36 (s, $4 \mathrm{H}$ from major), 3.70 $(\mathrm{s}, 3 \mathrm{H}), 3.57-3.61(\mathrm{~m}, 2.7 \mathrm{H}$ from THF), 3.22-3.32 (m, 0.77 $\mathrm{H}$ from major), 3.06 (s, br, $0.21 \mathrm{H}$ from minor), 2.94 (s, br, $0.62 \mathrm{H}$ from minor), 2.74 (s, b, 2.37 $\mathrm{H}$ from major), 2.31 $(\mathrm{s}, 2.39 \mathrm{H}$ from toluene), $2.03(\mathrm{t}, J=7.6 \mathrm{~Hz}, 3 \mathrm{H}), 1.56(\mathrm{~d}, J=11.6 \mathrm{~Hz}, 9 \mathrm{H}), 1.05(\mathrm{~d}, J=$ $11.6 \mathrm{~Hz}, 9 \mathrm{H}), 0.80-2.50(\mathrm{~m}, 22 \mathrm{Cy} \mathrm{H}) ;{ }^{31} \mathrm{P}\left\{{ }^{1} \mathrm{H}\right] \mathrm{NMR}\left(\mathrm{THF}-d_{8},-30{ }^{\circ} \mathrm{C}\right) 70.96(\mathrm{~d}, J=29.1$ $\mathrm{Hz}$, major), 68.74 (d, $J=27.5 \mathrm{~Hz}$, minor), 25.82 (d, $J=27.5 \mathrm{~Hz}$, minor), 20.12 (d, $J=$ 
$29.1 \mathrm{~Hz}$, major). IR: $v\left(\mathrm{NH}_{3}\right) 3088,3172,3255,3321,3364 \mathrm{~cm}^{-1}$. Anal. Calcd. For $\mathrm{C}_{40} \mathrm{H}_{62} \mathrm{~F}_{3} \mathrm{FeNO}_{4} \mathrm{P}_{2} \mathrm{Pd} \bullet 0.8$ equiv. of toluene: $\mathrm{C}, 54.34 ; \mathrm{H}, 6.83 ; \mathrm{N}, 1.39$. Found: $\mathrm{C}, 54.16$; $\mathrm{H}, 6.50 ; \mathrm{N}, 1.32$.

Preparation of (CyPF-t-Bu)Pd(4-MeOPh)(NH $\left.\mathbf{N H}_{2}\right) \quad$ (3). $\quad[(\mathrm{CyPF}-t-\mathrm{Bu}) \mathrm{Pd}(4-$ $\mathrm{MeOPh})\left(\mathrm{NH}_{3}\right)$ ]OTf $(147 \mathrm{mg}, 0.150 \mathrm{mmol})$ was dissolved in $5 \mathrm{~mL}$ of THF. $\mathrm{KN}\left(\mathrm{SiMe}_{3}\right)_{2}$ (33.0 $\mathrm{mg}, 0.160 \mathrm{mmol}$ ) was added to the stirred solution as a solid. The reaction was allowed to stir at room temperature for $30 \mathrm{~min}$, and it turned to a slightly pale yellowgreen color. The solvent was evaporated under vacuum. The resulting solid was dissolved in $3 \mathrm{~mL}$ benzene. The benzene solution was filtered through a plug of Celite, and benzene was evaporated under vacuum. The solid was dissolved in $1 \mathrm{~mL}$ THF and was layered with pentane. Pure yellow crystals $(47.0 \mathrm{mg}, 40 \%)$ were obtained by cooling the layered solution at $-35{ }^{\circ} \mathrm{C}$ for two days. ${ }^{1} \mathrm{H}$ NMR $\left(\mathrm{C}_{6} \mathrm{D}_{6}\right) \delta 7.88(\mathrm{br}, 1 \mathrm{H}), 7.64(\mathrm{br}, 1 \mathrm{H}), 7.04(\mathrm{~d}$, $J=7.2 \mathrm{~Hz}, 1 \mathrm{H}), 6.94(\mathrm{~d}, J=6.0 \mathrm{~Hz}, 1 \mathrm{H}), 4.56(\mathrm{~s}, \mathrm{br}, 1 \mathrm{H}), 4.06(\mathrm{~s}, \mathrm{br}, 1 \mathrm{H}), 4.01(\mathrm{~s}, 6$ H), 3.55-3.57 (m, 0.4 H from THF), $3.48(\mathrm{~s}, 3 \mathrm{H}), 3.00-3.10(\mathrm{~m}, 1 \mathrm{H}), 1.69(\mathrm{t}, J=6.8 \mathrm{~Hz}$, $3 \mathrm{H}), 1.56(\mathrm{~d}, J=10.8 \mathrm{~Hz}, 9 \mathrm{H}), 1.30(\mathrm{~d}, J=12.8 \mathrm{~Hz}, 9 \mathrm{H}), 0.80-2.5(\mathrm{~m}, 22 \mathrm{Cy} \mathrm{H}), 0.47$ (s, br, $2 \mathrm{H}) ;{ }^{31} \mathrm{P}\left\{{ }^{1} \mathrm{H}\right] \mathrm{NMR}\left(\mathrm{THF}-d 8,-30{ }^{\circ} \mathrm{C}\right) 65.03(\mathrm{~d}, J=31.9 \mathrm{~Hz}$, major), $64.74(\mathrm{~d}, J=$ $30.1 \mathrm{~Hz}$, minor), 17.98 (d, $J=30.8 \mathrm{~Hz}$, minor), 11.45 (d, $J=30.8 \mathrm{~Hz}$, major). IR: $v\left(\mathrm{NH}_{2}\right)$ $3278,3356 \mathrm{~cm}^{-1}$. Suitable elemental analysis was not obtained because the compound decomposes at room temperature as a solid over a $12-24 \mathrm{~h}$ time period.

Reductive elimination reaction from $(\mathrm{CyPF}-t-\mathrm{Bu}) \mathrm{Pd}(4-\mathrm{MeOPh})\left(\mathbf{N H}_{2}\right)$ 3. (CyPF- $t$ $\mathrm{Bu}) \mathrm{Pd}(4-\mathrm{MeOPh})\left(\mathrm{NH}_{2}\right)\left(8.2 \mathrm{mg}, 1.0 \times 10^{-3} \mathrm{mmol}\right)$ and $\mathrm{P}\left(\mathrm{Ph}-d_{5}\right)_{3}\left(2.7 \mathrm{mg}, 2.0 \times 10^{-3}\right.$ mmol) were dissolved $0.5 \mathrm{~mL} \mathrm{C}_{6} \mathrm{D}_{6}$ containing $1,3,5$-trimethoxybenzene $(0.29 \mathrm{mg}, 1.7 \times$ $\left.10^{-3} \mathrm{mmol}\right) . \mathrm{A}^{1} \mathrm{H}$ NMR spectrum was recorded. The tube was sealed and heated at $90{ }^{\circ} \mathrm{C}$ in a oil bath. ${ }^{31} \mathrm{P}\left\{{ }^{1} \mathrm{H}\right\}$ NMR spectrometry showed the disappearance of the starting material and the formation of $(\mathrm{CyPF}-t-\mathrm{Bu}) \mathrm{PdP}\left(\mathrm{Ph}-d_{5}\right)_{3}$. A ${ }^{1} \mathrm{H}$ NMR spectrum was recorded, and the yield of 4-tert-butylaniline and di(4-tert-butylphenyl)amine was determined by integrating the aromatic $\mathrm{C}-\mathrm{H}$ resonances of amines vs. the internal standard. 


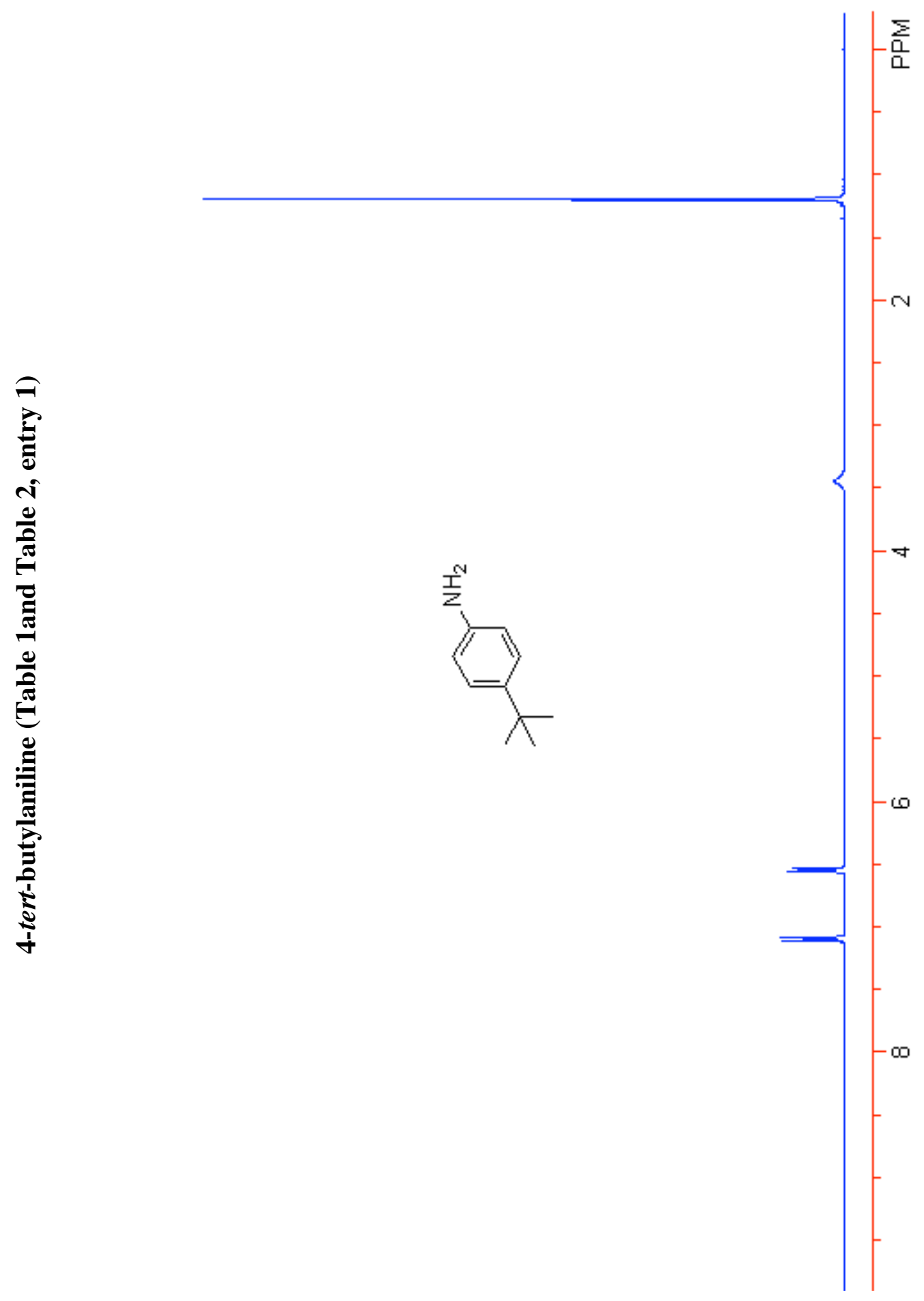




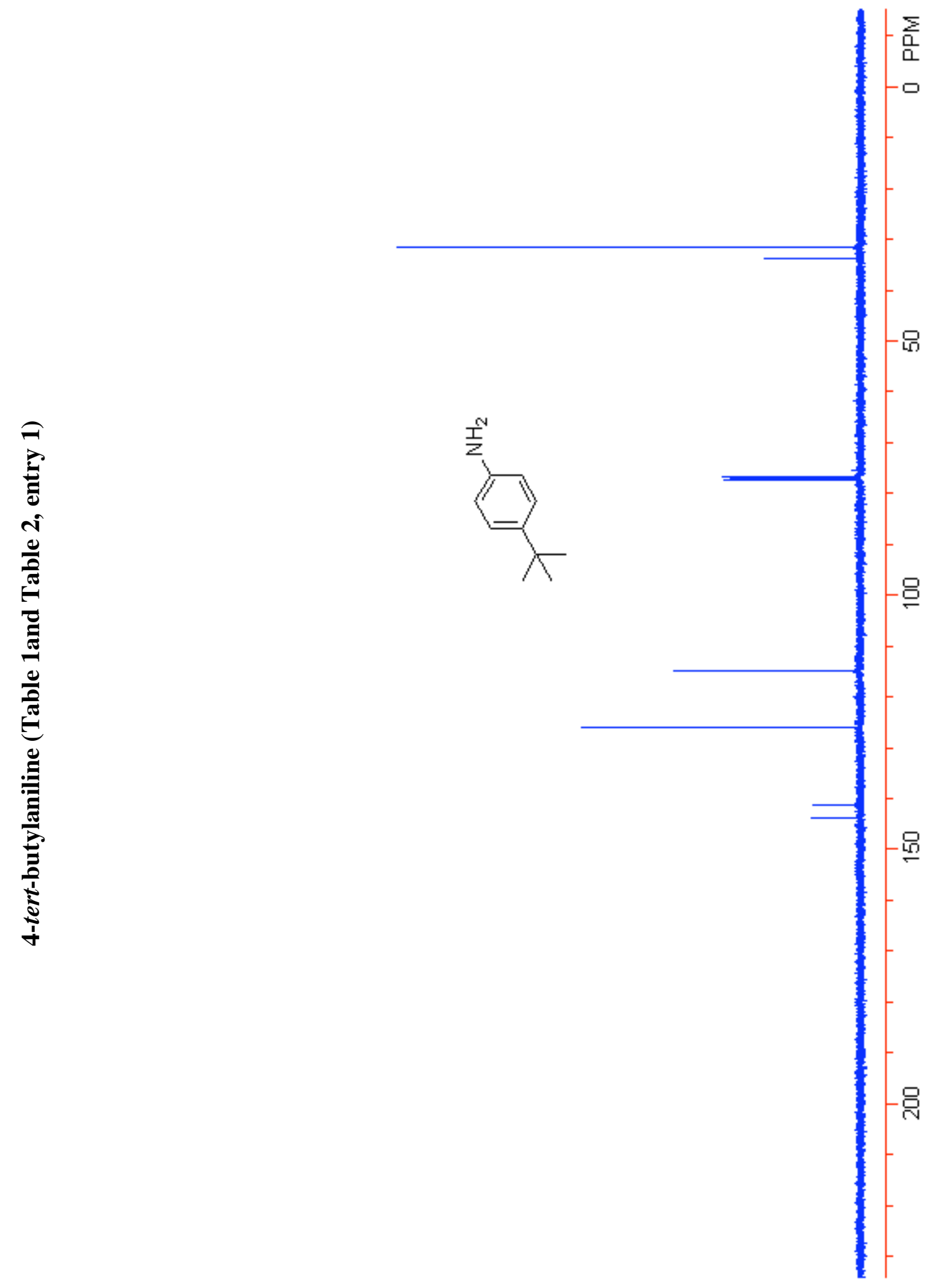




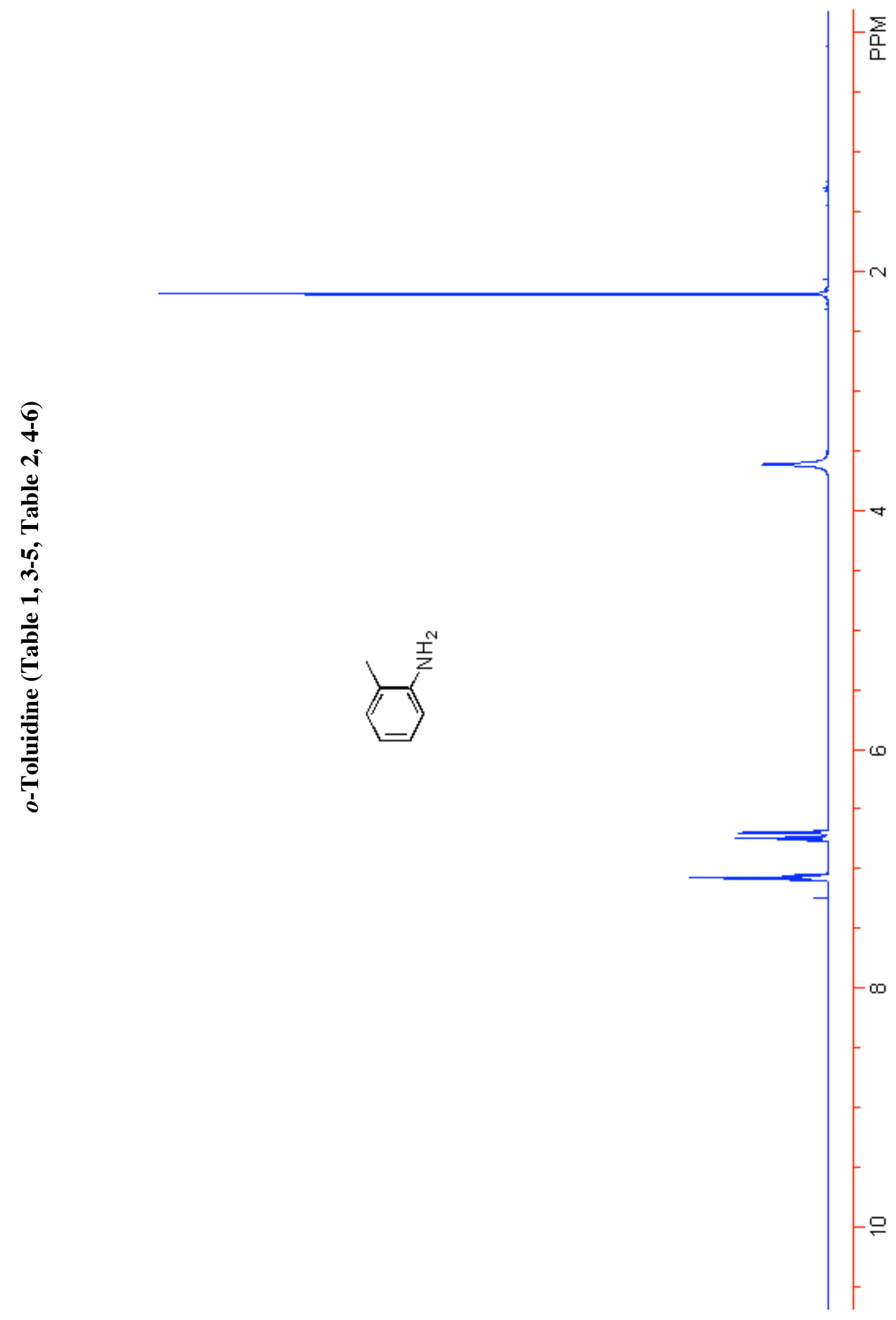




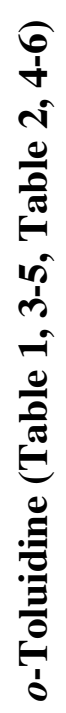

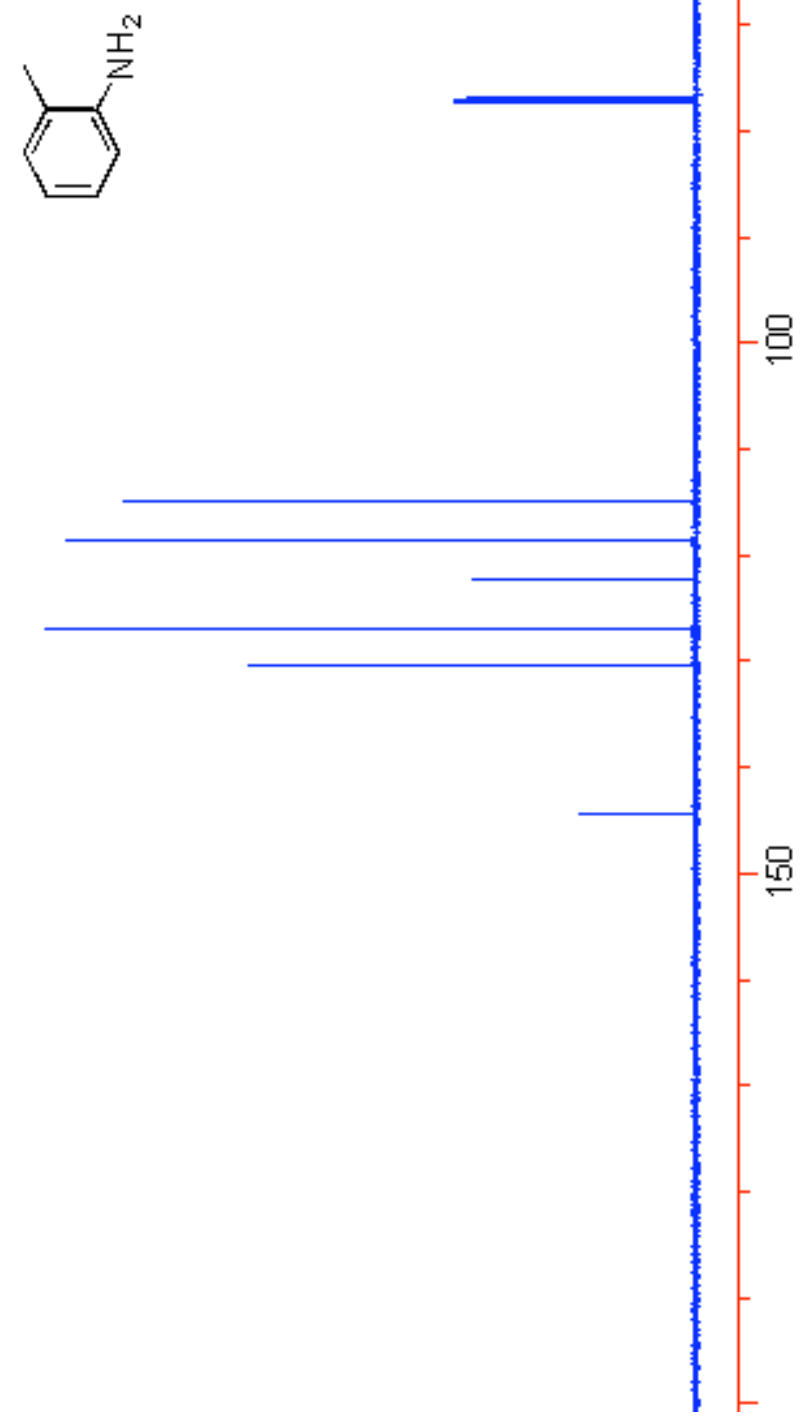



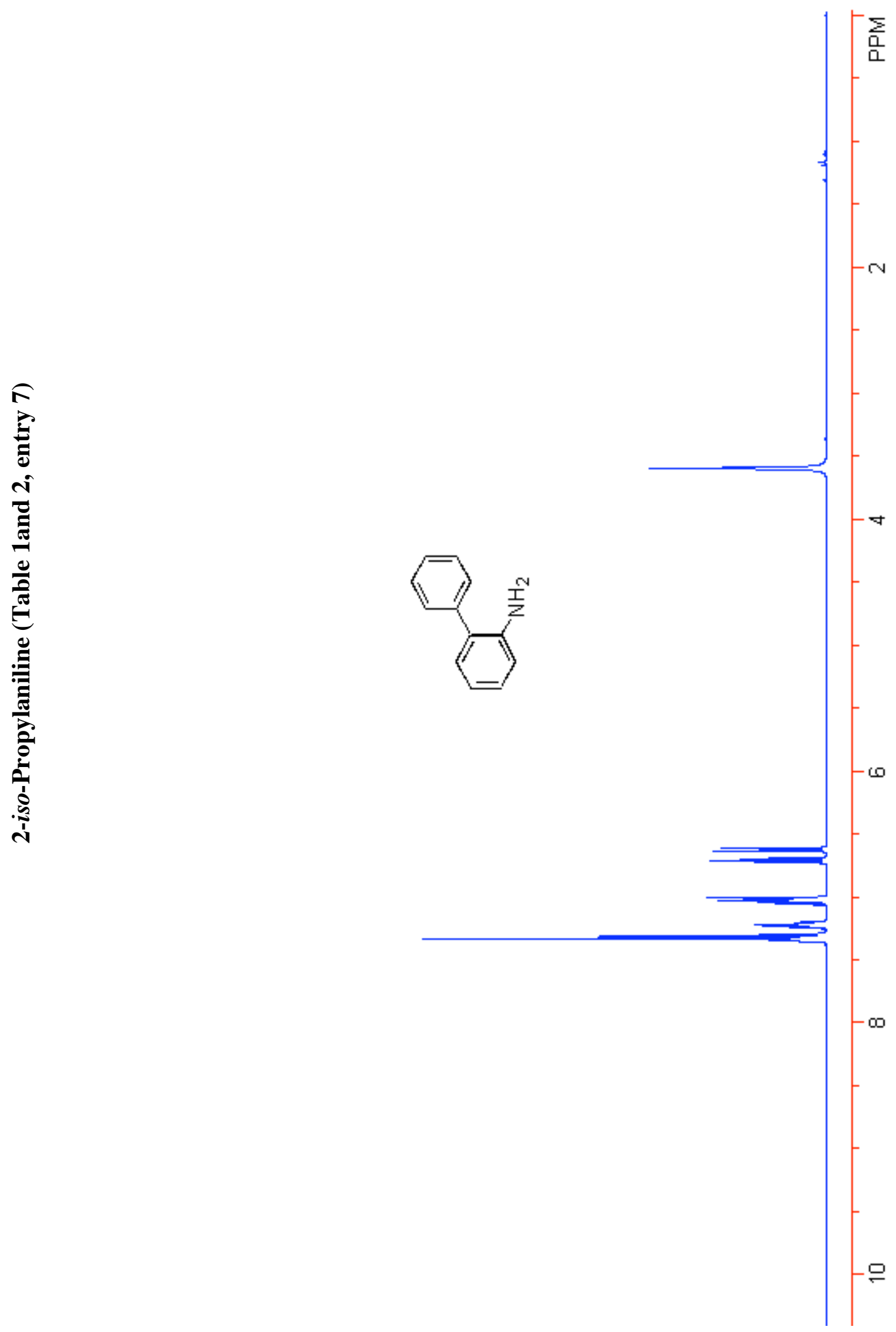


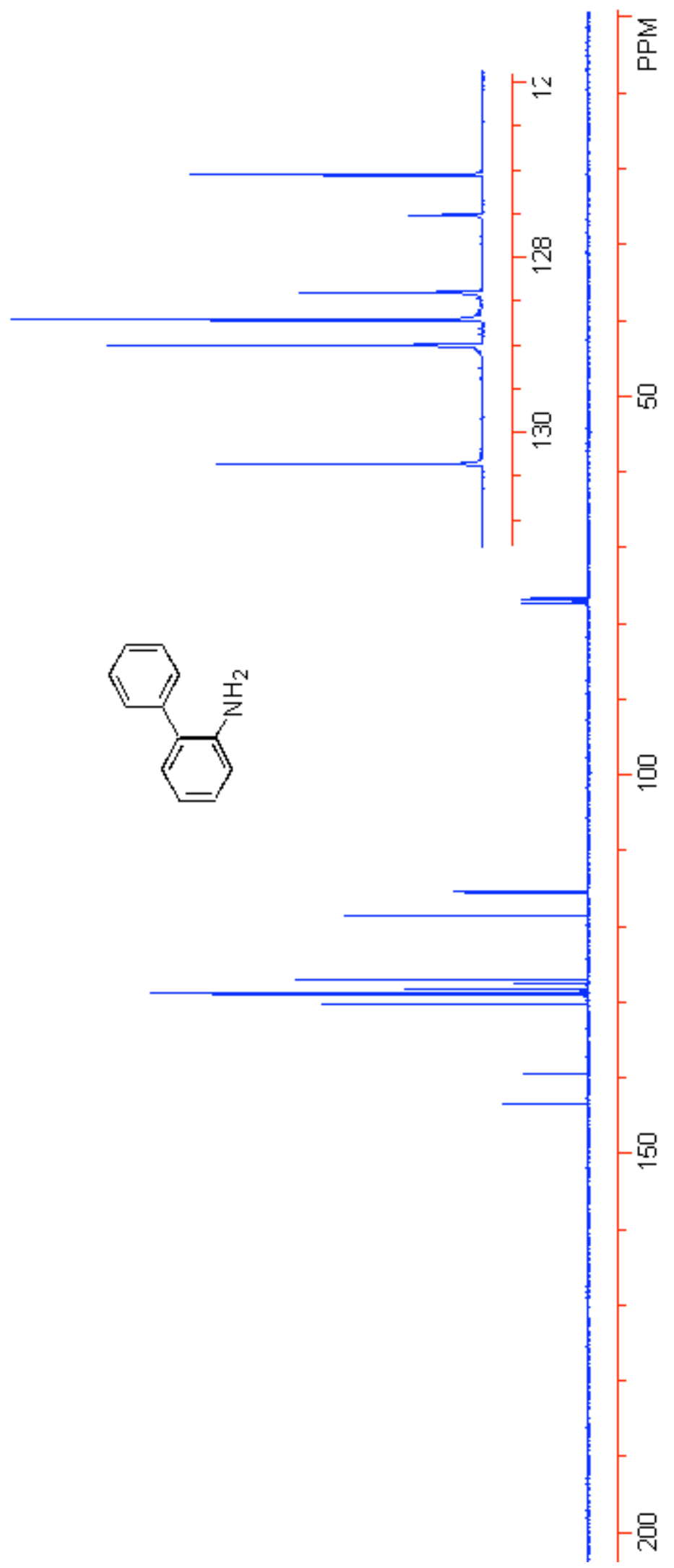




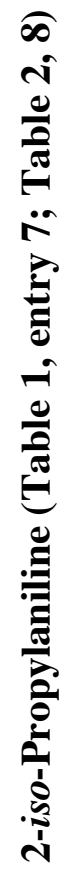

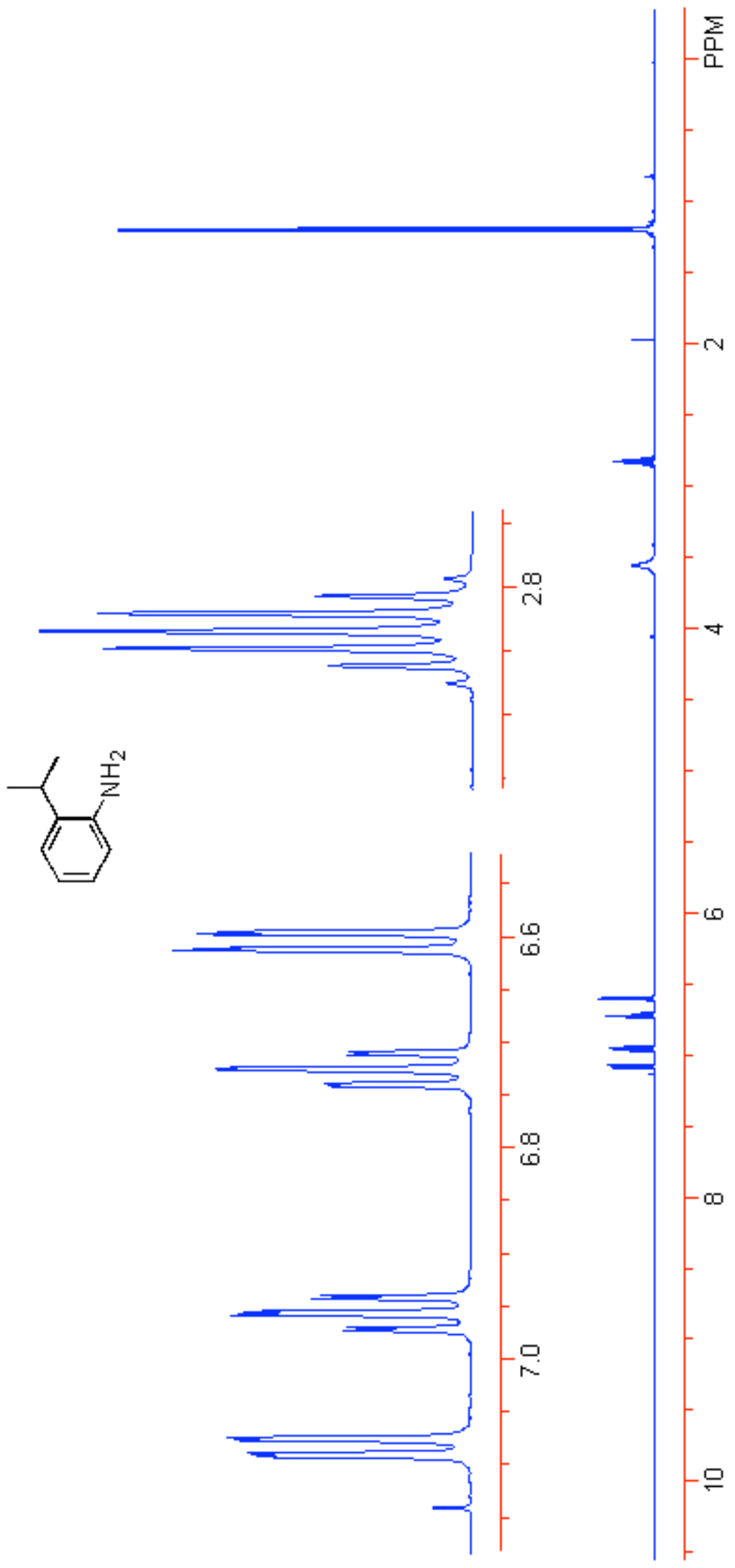




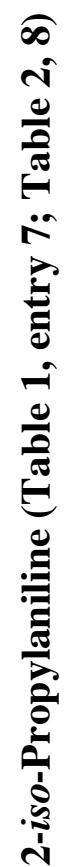

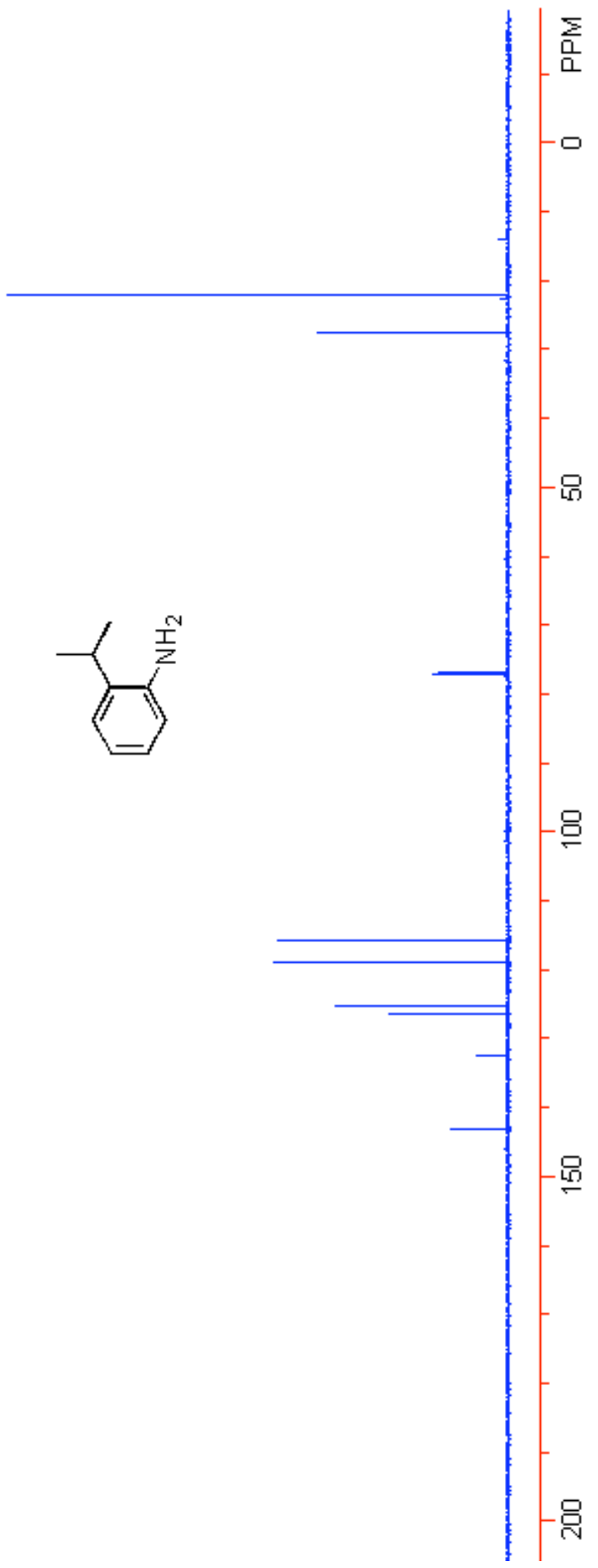



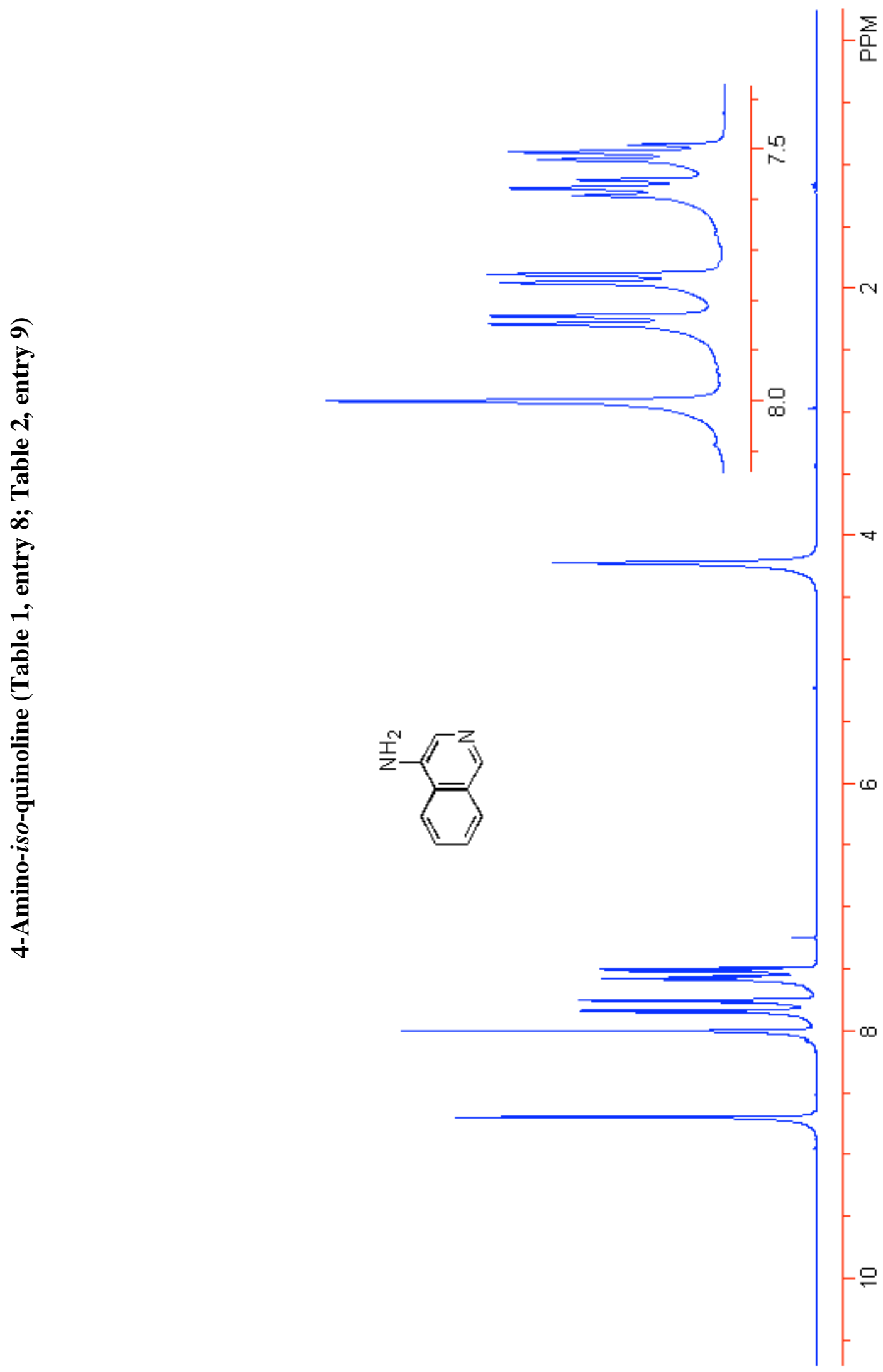


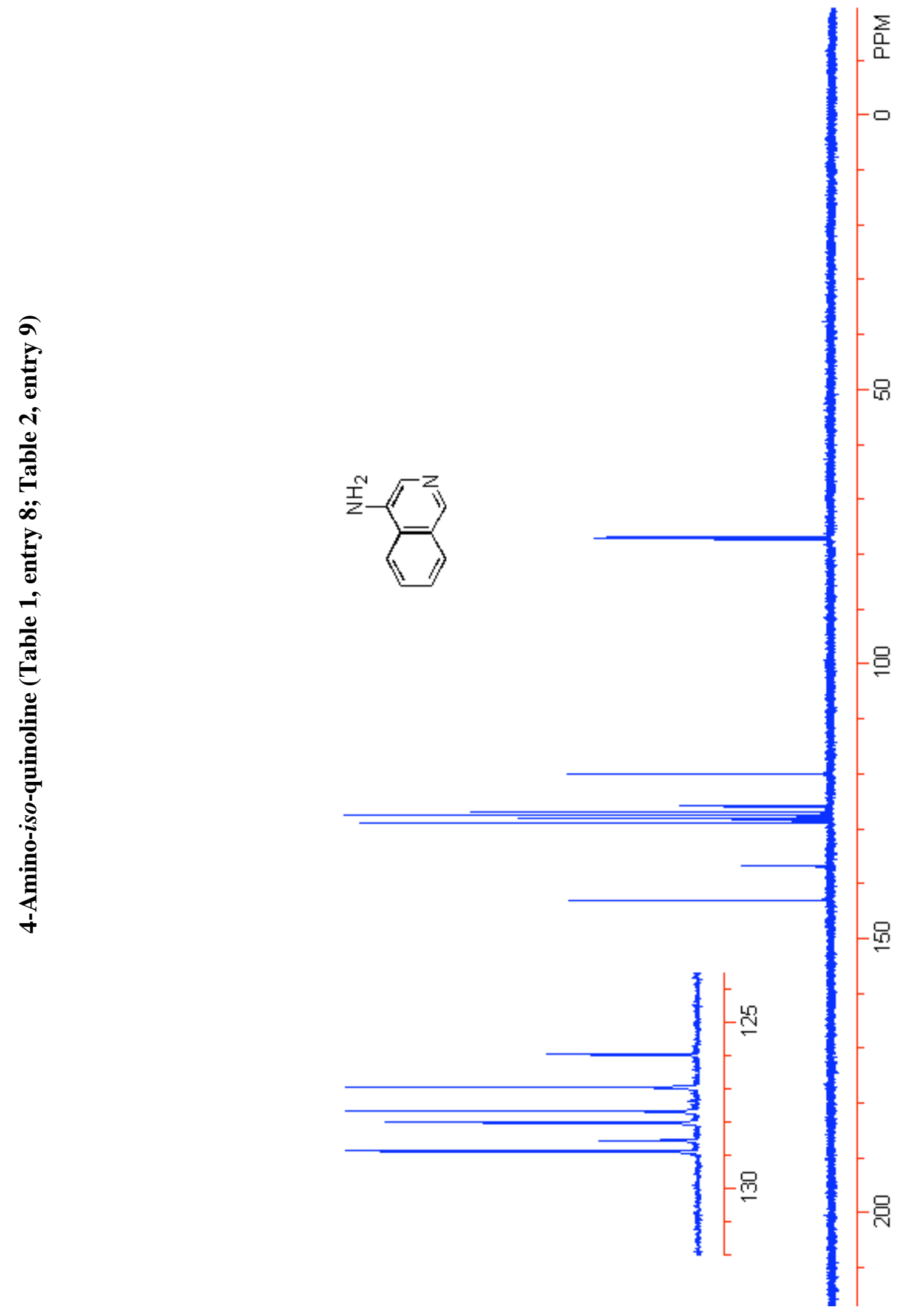




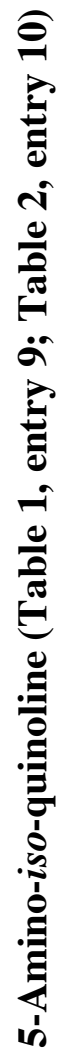

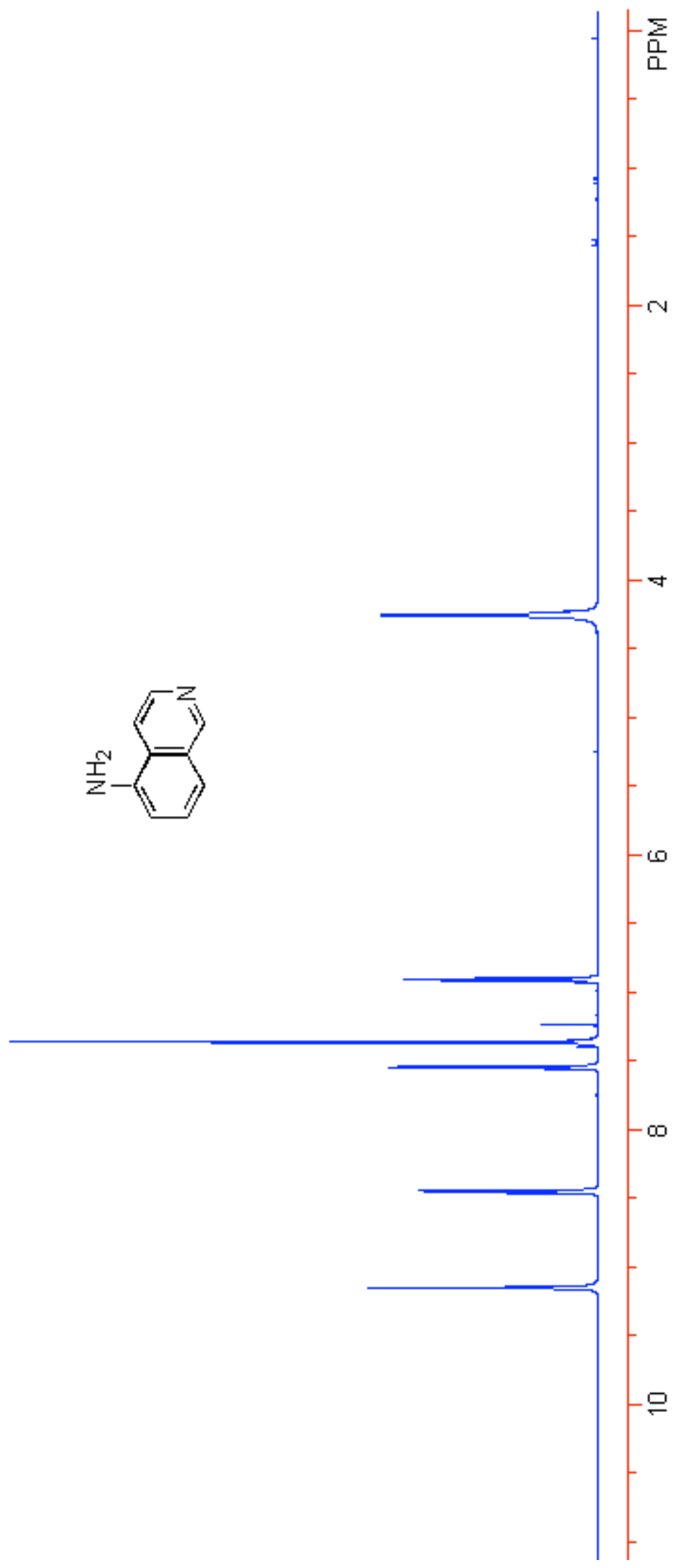




$$
11
$$




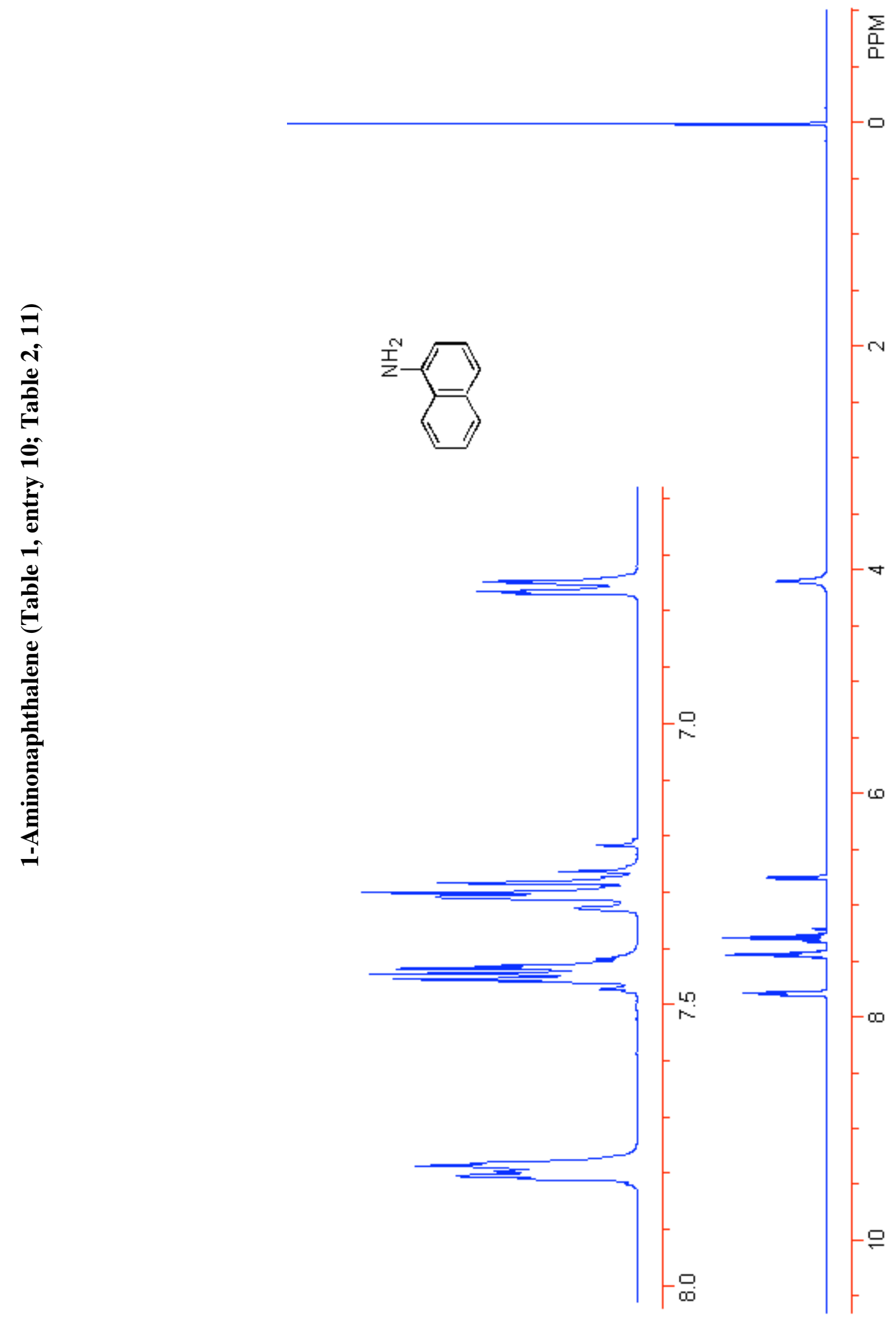




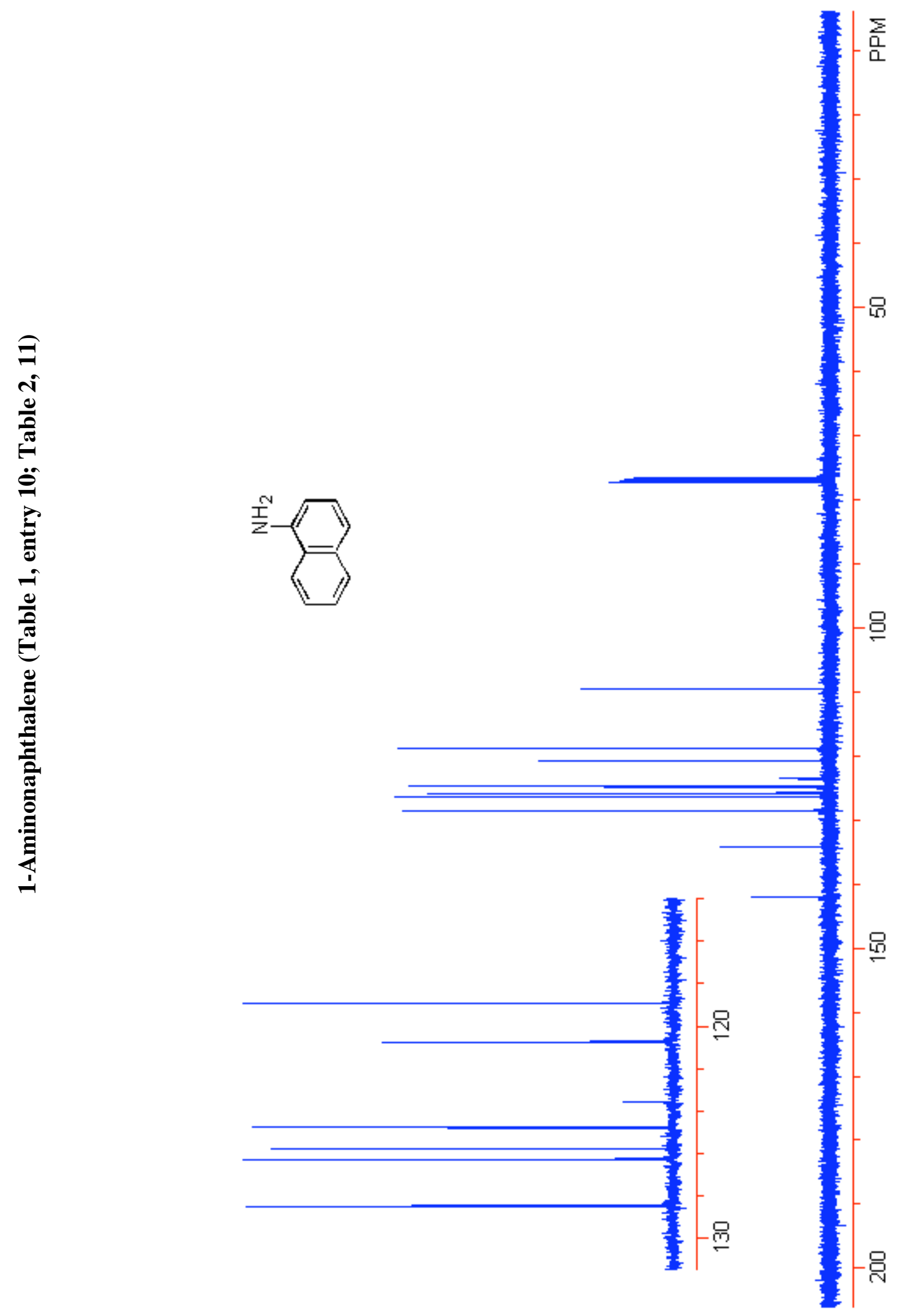




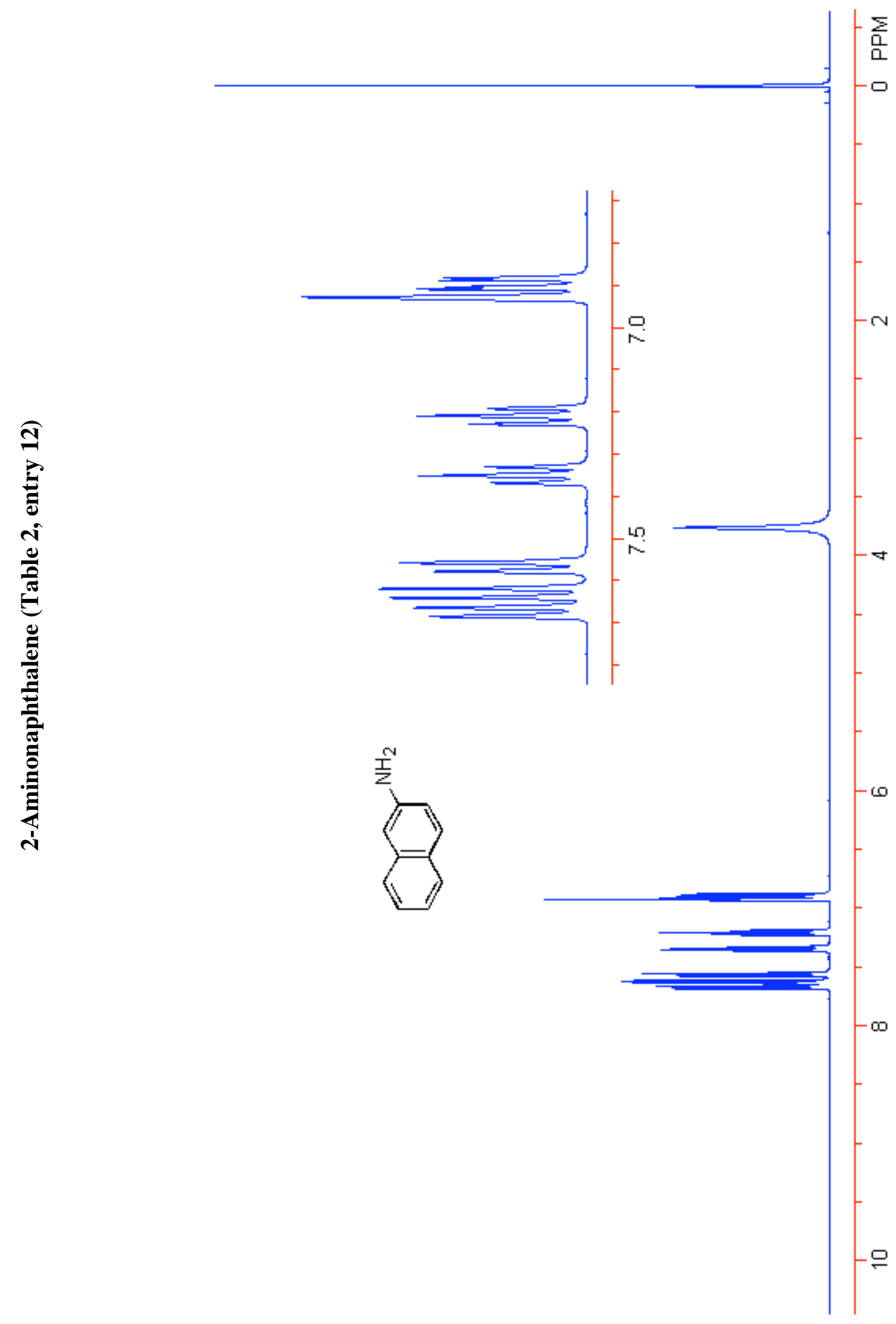




$$
\text { 1月 }
$$




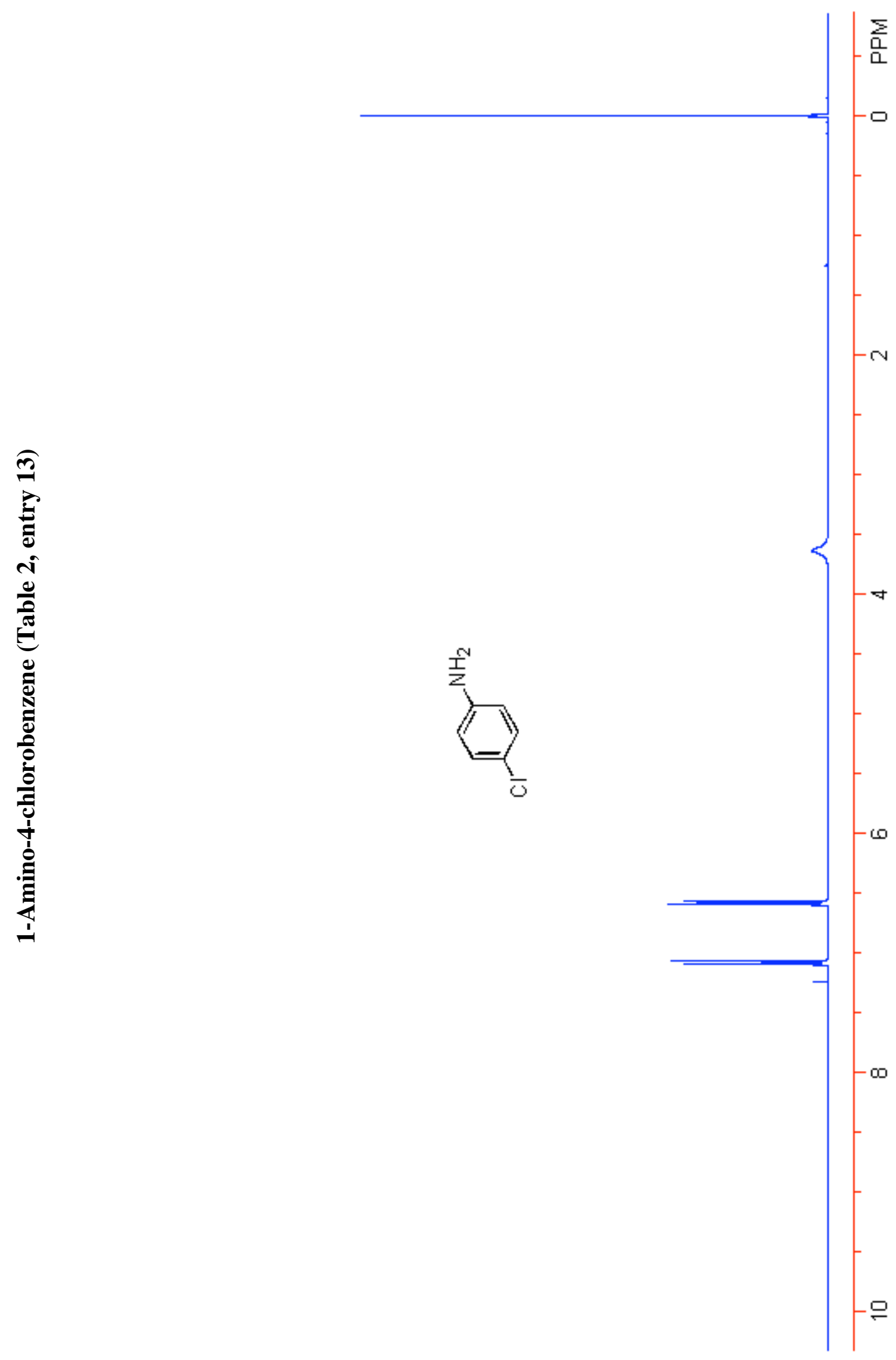




$$
1
$$




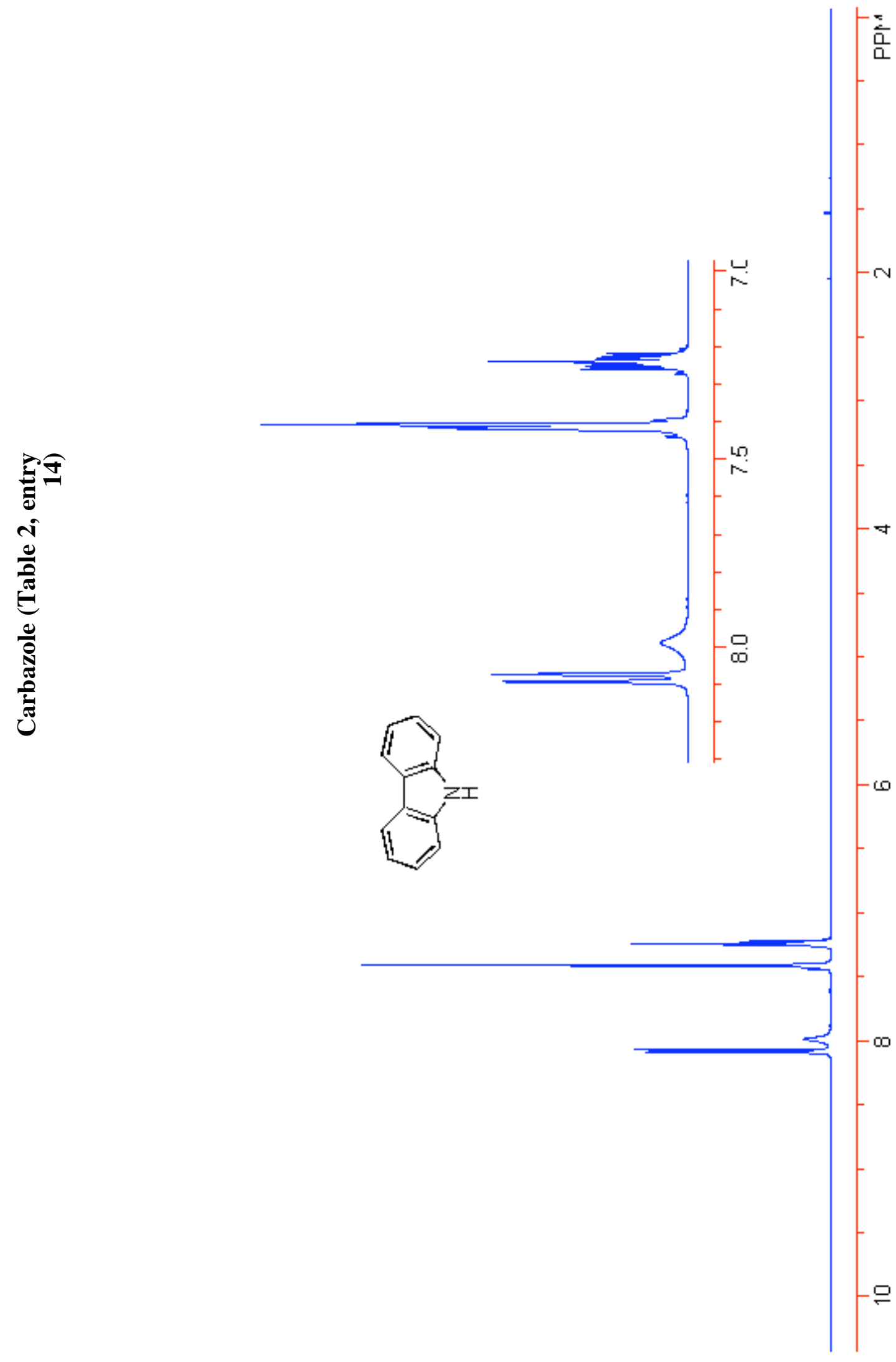



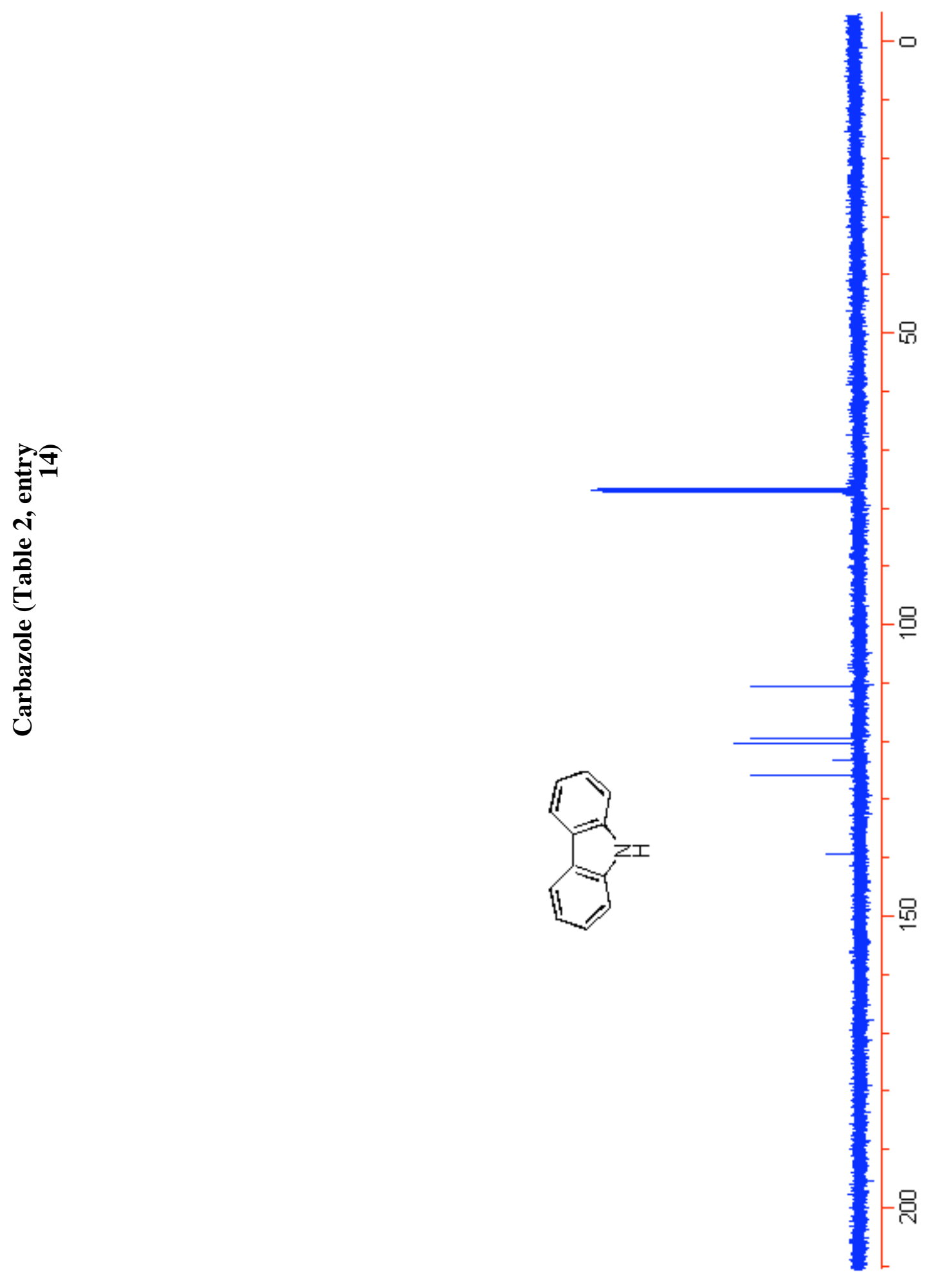


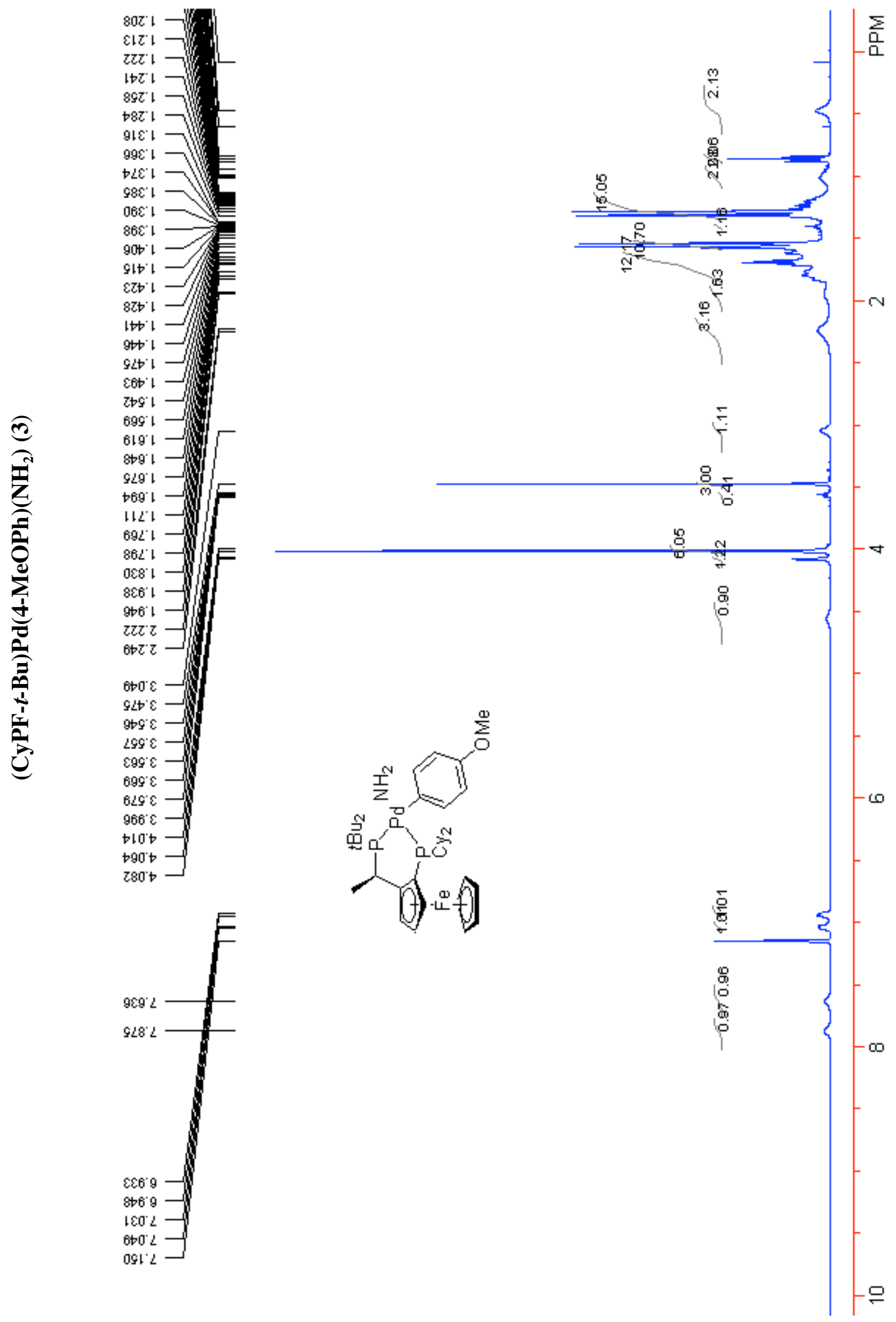




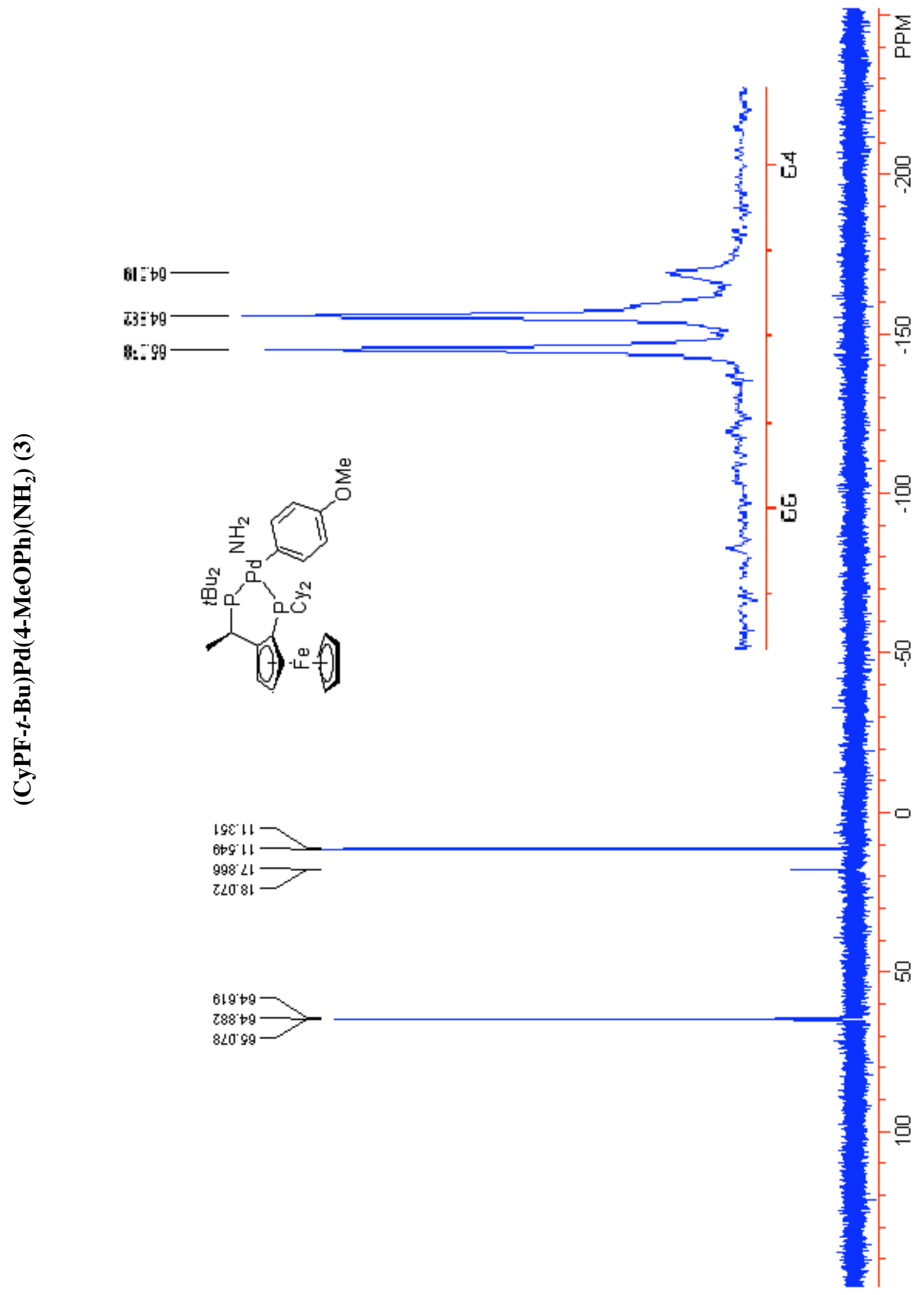

